\title{
ENDOSCOPY AND COHOMOLOGY IN A TOWER OF CONGRUENCE MANIFOLDS FOR $U(n, 1)$
}

\author{
SIMON MARSHALL ${ }^{1}$ and SUG WOO SHIN ${ }^{2,3}$ \\ ${ }^{1}$ Department of Mathematics, University of Wisconsin - Madison, \\ 480 Lincoln Drive, Madison, WI 53706, USA; \\ email:marshall@math.wisc.edu \\ 2 Department of Mathematics, University of California, Berkeley, \\ 901 Evans Hall, Berkeley, CA 94720, USA; \\ email: sug.woo.shin@ berkeley.edu \\ ${ }^{3}$ Korea Institute for Advanced Study, Dongdaemun-gu, Seoul 130-722, Republic of Korea
}

Received 13 April 2019; accepted 2 May 2019

\begin{abstract}
By assuming the endoscopic classification of automorphic representations on inner forms of unitary groups, which is currently work in progress by Kaletha, Minguez, Shin, and White, we bound the growth of cohomology in congruence towers of locally symmetric spaces associated to $U(n, 1)$. In the case of lattices arising from Hermitian forms, we expect that the growth exponents we obtain are sharp in all degrees.
\end{abstract}

2010 Mathematics Subject Classification: 32N10 (primary); 32M15 (secondary)

\section{Introduction}

This paper studies the limit multiplicity problem for cohomological automorphic forms on arithmetic quotients of $U(N-1,1)$. Let $F$ be a totally real number field with ring of integers $\mathcal{O}_{F}$. Write $\mathbb{A}$ for the ring of adeles over $F$. Let $U(N)=$ $U_{E / F}(N)$ denote the quasisplit unitary group with respect to a totally imaginary quadratic extension $E$ of $F$. Let $G$ be a unitary group over $F$ that is an inner form of $U_{E / F}(N)$. We assume that $G$ has signature $(N-1,1)$ at one real place and compact factors at all other real places. Let $S$ be a finite set of places to be defined later, and which includes all infinite places, and let $\mathfrak{n} \subset \mathcal{O}_{F}$ be a nonzero ideal that is divisible only by primes away from $S$ that split in $E / F$. We let $K(\mathfrak{n}) \subset G\left(\mathbb{A}_{f}\right)$

(c) The Author(s) 2019. This is an Open Access article, distributed under the terms of the Creative Commons Attribution licence (http://creativecommons.org/licenses/by/4.0/), which permits unrestricted re-use, distribution, and reproduction in any medium, provided the original work is properly cited. 
be the compact congruence subgroup of level $\mathfrak{n}$, and let $\Gamma(\mathfrak{n})=G(F) \cap K(\mathfrak{n})$ be the congruence arithmetic lattice in $U(N-1,1)$ of level $\mathfrak{n}$ associated to $G$. Let $Y(\mathfrak{n})$ be the manifold $\Gamma(\mathfrak{n}) \backslash U(N-1,1) / U(N-1) \times U(1)$, which is a connected finite volume complex hyperbolic manifold of complex dimension $N-1$. (See equation (12) for the precise definition.) Write $h_{(2)}^{d}(Y(\mathfrak{n}))$ for the dimension of the $L^{2}$-cohomology of $Y(\mathfrak{n})$ in degree $d \geqslant 0$.

THEOREM 1.1. Assume the endoscopic classification for inner forms of $U(N)$ stated in [16, Theorem 1.7.1]. If $d<N-1$, we have

$$
h_{(2)}^{d}(Y(\mathfrak{n})) \ll_{\epsilon} \operatorname{vol}(Y(\mathfrak{n}))^{N d /\left(N^{2}-1\right)+\epsilon} .
$$

The case $d>N-1$ follows by Poincare duality. It is well known that $h_{(2)}^{N-1}(Y(\mathfrak{n})) \sim \operatorname{vol}(Y(\mathfrak{n}))$. Previous results of this type in the case of $U(2,1)$ and $U(2,2)$ can be found in work of the first author [19, 20]; see also [12] for the case of $U(N-1,1)$.

The introduction of [16] clarifies the conditionality of [16, Theorem 1.7.1]. In a nutshell, we are still waiting for the remaining case in Chaudouard-Laumon's proof of the weighted fundamental lemma and the papers [A25,A26] as cited in [1]. In addition we need the sequel papers [KMSb] and [KMSa] (still in preparation) as cited in [16] to complete the proof of Theorem 1.7.1 for pure inner forms and all inner forms of $U(N)$, respectively. In the meantime, the stabilization of the twisted trace formula has been completed by MoeglinWaldspurger [23, 24], so this is no longer an obstacle.

Theorem 1.1 fits into the general framework of estimating the asymptotic multiplicities of automorphic forms. We now recall the general formulation of this problem, and some of the previous results on it. Let $G$ be a semisimple real algebraic group with no compact factors. We still write $G$ for $G(\mathbb{R})$, the real group of $\mathbb{R}$-points, if there is no danger of confusion. If $\Gamma \subset G$ is a lattice and $\pi$ an irreducible unitary representation of $G$, we let $m(\pi, \Gamma)$ be the multiplicity with which $\pi$ appears in $L^{2}(\Gamma \backslash G)$. If we now assume that $\Gamma$ is congruence arithmetic and that $\Gamma_{n} \subset \Gamma$ is a family of principal congruence subgroups, the limit multiplicity problem is to provide estimates for $m\left(\pi, \Gamma_{n}\right)$.

A general principle that has emerged from work on this problem is that, the further $\pi$ is from being discrete series, the better bounds one should be able to prove for $m\left(\pi, \Gamma_{n}\right)$. If we define $V(n)=\operatorname{vol}\left(\Gamma_{n} \backslash G\right)$, the trivial bound (at least when $\Gamma$ is cocompact) is $m\left(\pi, \Gamma_{n}\right) \ll V(n)$, and it is known from work of de George and Wallach [13] (if $\Gamma$ is cocompact) and Savin [29] (if it is not) that this is realized if and only if $\pi$ is in the discrete series. In the cocompact case, it also follows from [13] that if $\pi$ is nontempered, then one has a bound of the 
form $m\left(\pi, \Gamma_{n}\right) \ll V(n)^{1-\delta(\pi)}$ for some $\delta(\pi)>0$; see the introduction of [28] for an explanation of this principle, and [33] for an explicit determination of such a $\delta(\pi)$ in some cases.

For the most highly nontempered representation, namely the trivial one, one has $m\left(\pi, \Gamma_{n}\right)=1$. Sarnak and Xue [28] made a conjecture that interpolates between this and $m\left(\pi, \Gamma_{n}\right) \ll V(n)$ in the discrete series case. Define $p(\pi)$ to be the infimum over $p$ for which the $K$-finite matrix coefficients of $\pi$ lie in $L^{p}(G)$. We then have:

Conjecture 1 (Sarnak-Xue). For fixed $\pi$, we have $m\left(\pi, \Gamma_{n}\right) \ll_{\epsilon} V(n)^{2 / p(\pi)+\epsilon}$.

Note that Conjecture 1 is weaker than the trivial bound in both cases of $\pi$ discrete or trivial. The point is that it is much stronger for general nontempered $\pi$ than what one can prove using the methods of de George-Wallach mentioned above. Sarnak and Xue established Conjecture 1 for $\operatorname{SL}(2, \mathbb{R})$ or $\operatorname{SL}(2, \mathbb{C})$, and proved an approximation for $\mathrm{SU}(2,1)$ that, in our setting, implies that $h^{1}(Y(\mathfrak{n})) \ll \operatorname{vol}(Y(\mathfrak{n}))^{7 / 12+\epsilon}$ when $N=3$ and $\Gamma$ is cocompact and arises from a Hermitian form.

We show in Proposition 3.1 that the representations $\pi$ of $U(N-1,1)$ contributing to $h_{(2)}^{d}(Y(\mathfrak{n}))$ all have $p(\pi) \geqslant 2(N-1) / d$. In the setting of Theorem 1.1 , Conjecture 1 therefore predicts that $h_{(2)}^{d}(Y(\mathfrak{n})) \ll_{\epsilon} \operatorname{vol}(Y(\mathfrak{n}))^{d /(N-1)+\epsilon}$, so that Theorem 1.1 in fact represents a strengthening of this conjecture.

We note that there has also been significant progress recently on the problem of showing that the normalized discrete spectral measure of $L^{2}\left(\Gamma_{n} \backslash G\right)$ tends weakly to the Plancherel measure of $G$. This work is in some sense orthogonal to ours, and as formulated these results do not provide information on $m\left(\pi, \Gamma_{n}\right)$ beyond showing that $m\left(\pi, \Gamma_{n}\right) / V(n)$ approaches the expected value.

1.1. Outline of the proof. We go back to the unitary group $G$ over $F$ introduced at the very beginning. Let $K_{\infty}$ denote a maximal compact subgroup of $G\left(F \otimes_{\mathbb{Q}} \mathbb{R}\right)$ so that $K_{\infty}$ is isomorphic to $U(N-1) \times U(1) \times U(N)^{[F: \mathbb{Q}]-1}$. It will be more convenient for us to work on the possibly disconnected arithmetic quotients $X(\mathfrak{n})=G(F) \backslash G(\mathbb{A}) / K(\mathfrak{n}) K_{\infty}$. If $q_{v}$ denotes the order of the residue field of $F_{v}$, we prove the following more precise bound.

THEOREM 1.2. Assume the endoscopic classification for inner forms of $U(N)$ stated in [16, Theorem 1.7.1]. If $d<N-1$, we have

$$
h_{(2)}^{d}(X(\mathfrak{n})) \ll \prod_{v \mid \mathfrak{n}}\left(1-1 / q_{v}\right) N \mathfrak{n}^{N d+1},
$$

except when $N=4$ and $d=2$ when we have $h_{(2)}^{d}(X(\mathfrak{n})) \ll_{\epsilon} N \mathfrak{n}^{N d+1+\epsilon}$. 
Theorem 1.1 follows from this, as $X(\mathfrak{n})$ contains $\gg_{\epsilon} N \mathfrak{n}^{1-\epsilon}$ copies of $Y(\mathfrak{n})$ and we have $\operatorname{vol}(Y(\mathfrak{n}))=N \mathfrak{n}^{N^{2}-1+o(1)}$. We now give an outline of the proof of Theorem 1.2. For simplicity, we shall either omit or simplify much of the notation for things like Arthur parameters and packets. Because of this, all notation introduced here is temporary. (Refer to Section 2 for unexplained notation.)

Let $\Phi_{\text {sim }}(n)$ denote the set of conjugate self-dual cusp forms on $\operatorname{GL}\left(n, \mathbb{A}_{E}\right)$, and let $v(l)$ denote the unique irreducible (complex algebraic) representation of $\operatorname{SL}(2, \mathbb{C})$ of dimension $l$. Let $U(n)$ be the quasisplit unitary group of degree $n$ with respect to $E / F$. Let $\Psi_{2}(n)$ denote the set of square-integrable Arthur parameters for $U(n)$, which are formal sums $\psi=\phi_{1} \otimes v\left(n_{1}\right) \boxplus \cdots \boxplus \phi_{k} \otimes v\left(n_{k}\right)$ with $\phi_{i} \in \Phi_{\text {sim }}\left(m_{i}\right)$, subject to certain conditions including that $n=\sum_{i \geqslant 1} n_{i} m_{i}$ and that the pairs $\phi_{i} \otimes v\left(n_{i}\right)$ have to be distinct. Any $\phi \in \Phi_{\text {sim }}(n)$ (respectively $\psi \in \Psi_{2}(n)$ ) has localizations $\phi_{v}$ (respectively $\psi_{v}$ ), which are local Langlands parameters (respectively Arthur parameters) for $U(n)$. To each $\psi \in \Psi_{2}(N)$ and each place $v$ of $F$, there is associated a local packet $\Pi_{\psi_{v}}(G)$ of representations of $G\left(F_{v}\right)$, and a global packet $\Pi_{\psi}(G)=\prod \Pi_{\psi_{v}}(G)$. If $\psi \in \Psi_{2}(N)$ and $K \subset G\left(\mathbb{A}_{f}\right)$ we define $\operatorname{dim}_{G}(K, \psi)=\sum_{\pi \in \Pi_{\psi}(G)} \operatorname{dim}\left(\pi_{f}^{K}\right)$. Similarly, one may associate to $\psi \in \Psi_{2}(n)$ a packet $\Pi_{\psi}(U(n))=\prod \Pi_{\psi_{v}}(U(n))$ for $U(n, \mathbb{A})$, and we define $\operatorname{dim}_{U(n)}(K, \psi)$ for $K \subset U\left(n, \mathbb{A}_{f}\right)$ analogously to $\operatorname{dim}_{G}(K, \psi)$.

The main result of the endoscopic classification implies that the automorphic spectrum of $G$ is contained in the union of $\Pi_{\psi}(G)$ for $\psi \in \Psi_{2}(N)$. If we combine this classification with Matsushima's formula, we have

$$
h_{(2)}^{d}(X(\mathfrak{n})) \leqslant \sum_{\psi \in \Psi_{2}(N)} \sum_{\pi \in \Pi_{\psi}(G)} \operatorname{dim} H^{d}\left(\mathfrak{g}, K_{\infty} ; \pi_{\infty}\right) \operatorname{dim} \pi_{f}^{K(\mathfrak{n})}
$$

where $H^{d}\left(\mathfrak{g}, K_{\infty} ; \pi_{\infty}\right)$ denotes the relative Lie algebra cohomology of $\pi_{\infty}$ as in [6]. The main part of the proof involves using the structure of the packets $\Pi_{\psi}(G)$ to bound the right hand side of (1) in terms of global multiplicities on smaller quasisplit unitary groups, which we then bound using a theorem of Savin. The key fact that allows us to control the power of $N \mathfrak{n}$ we obtain is due to Bergeron, Millson, and Moeglin [3, Proposition 13.2], and essentially states that if there exists $\pi \in \Pi_{\psi}(G)$ with $H^{d}\left(\mathfrak{g}, K_{\infty} ; \pi_{\infty}\right) \neq 0$, then $\psi$ must contain a representation $v(n)$ with $n \geqslant N-d$.

We define a shape to be a list of pairs $\left(n_{1}, m_{1}\right), \ldots,\left(n_{k}, m_{k}\right)$ with $\sum_{i \geqslant 1} m_{i} n_{i}=$ $N$, and may naturally talk about the shape of an Arthur parameter. If $\mathcal{S}=\left(n_{1}\right.$, $\left.m_{1}\right), \ldots,\left(n_{k}, m_{k}\right)$ is a shape, we let $\Psi_{2}(N)_{\mathcal{S}} \subset \Psi_{2}(N)$ be the set of parameters having that shape. If $\psi \in \Psi_{2}(N)_{\mathcal{S}}$, we let $\phi_{i} \in \Phi_{\text {sim }}\left(m_{i}\right)$ be the terms in the decomposition $\psi=\phi_{1} \otimes v\left(n_{1}\right) \boxplus \cdots \boxplus \phi_{k} \otimes v\left(n_{k}\right)$. We also define $P_{\mathcal{S}}$ to be the 
standard parabolic in $\mathrm{GL}_{N}$ of type

$$
(\underbrace{m_{1}, \ldots, m_{1}}_{n_{1} \text { times }}, \ldots, \underbrace{m_{k}, \ldots, m_{k}}_{n_{k} \text { times }}) .
$$

We now fix $\mathcal{S}$, and bound the contribution to (1) from $\Psi_{2}(N)_{\mathcal{S}}$, which we denote $h_{(2)}^{d}(X(\mathfrak{n}))_{\mathcal{S}}$. As mentioned above, we may assume that $n_{1} \geqslant N-d$. We may restrict our attention to those $\psi \in \Psi_{2}(N)_{\mathcal{S}}$ for which there is $\pi \in \Pi_{\psi}(G)$ with $H^{d}\left(\mathfrak{g}, K_{\infty} ; \pi_{\infty}\right) \neq 0$. This condition restricts $\psi_{\infty}$, and hence $\phi_{i, \infty}$, to finite sets which we denote $\Psi_{\infty}$ and $\Phi_{i, \infty}$, so that

$$
h_{(2)}^{d}(X(\mathfrak{n}))_{\mathcal{S}} \ll \sum_{\substack{\psi \in \Psi_{2}(N)_{\mathcal{S}} \\ \psi_{\infty} \in \Psi_{\infty}}} \operatorname{dim}_{G}(K(\mathfrak{n}), \psi) .
$$

In Section 5 we prove Proposition 5.1, which states that if the principal congruence subgroups $K_{i}(\mathfrak{n}) \subset U\left(m_{i}, \mathbb{A}_{f}\right)$ are chosen correctly, then one can bound $\operatorname{dim}_{G}(K(\mathfrak{n}), \psi)$ in terms of $\operatorname{dim}_{U\left(m_{i}\right)}\left(K_{i}(\mathfrak{n}), \phi_{i}\right)$. For most choices of $\mathcal{S}$, this bound has the form

$$
\operatorname{dim}_{G}(K(\mathfrak{n}), \psi) \ll N \mathfrak{n}^{\operatorname{dim~GL} / P_{\mathcal{S}}+\epsilon} \prod_{i=1}^{k} \operatorname{dim}_{U\left(m_{i}\right)}\left(K_{i}(\mathfrak{n}), \phi_{i}\right)^{n_{i}}
$$

We prove this bound by factorizing both sides over places of $F$. At nonsplit places we apply the trace identities that appear in the definition of the local packets $\Pi_{\psi_{v}}(G)$. At split places, $\Pi_{\psi_{v}}(G)$ is a singleton $\left\{\pi_{v}\right\}$, and we use the description of $\pi_{v}$ as the Langlands quotient of a representation induced from $P_{\mathcal{S}}$.

We next sum the bound (2) over $\psi \in \Psi_{2}(N)_{\mathcal{S}}$, or equivalently we sum $\phi_{i}$ over $\Phi_{\text {sim }}\left(m_{i}\right)$, which gives

$$
\begin{aligned}
h_{(2)}^{d}(X(\mathfrak{n}))_{\mathcal{S}} & \ll N \mathfrak{n}^{\operatorname{dim} \mathrm{GL}_{N} / P_{\mathcal{S}}+\epsilon} \prod_{i=1}^{k} \sum_{\substack{\phi_{i} \in \Phi_{\text {sim }}\left(m_{i}\right) \\
\phi_{i, \infty} \in \Phi_{i, \infty}}} \operatorname{dim}_{U\left(m_{i}\right)}\left(K_{i}(\mathfrak{n}), \phi_{i}\right)^{n_{i}} \\
& \leqslant N \mathfrak{n}^{\operatorname{dim} \mathrm{GL}_{N} / P_{\mathcal{S}}+\epsilon} \prod_{i=1}^{k}\left(\sum_{\substack{\phi_{i} \in \Phi_{\operatorname{sim}}\left(m_{i}\right) \\
\phi_{i, \infty} \in \Phi_{i, \infty}}} \operatorname{dim}_{U\left(m_{i}\right)}\left(K_{i}(\mathfrak{n}), \phi_{i}\right)\right)^{n_{i}} .
\end{aligned}
$$

If we define $\Theta_{i, \infty}$ to be the union of $\Pi_{\phi_{i, \infty}}\left(U\left(m_{i}\right)\right)$ over $\phi_{i, \infty} \in \Phi_{i, \infty}$, then $\Theta_{i, \infty}$ is finite. Moreover, because the parameters $\phi_{i}$ are simple generic, the packet $\Pi_{\phi_{i}}\left(U\left(m_{i}\right)\right)$ is stable, so all representations in it occur discretely on $U\left(m_{i}\right)$. This implies that

$$
\sum_{\substack{\phi_{i} \in \Phi_{\operatorname{sim}}\left(m_{i}\right) \\ \phi_{i, \infty} \in \Phi_{i, \infty}}} \operatorname{dim}_{U\left(m_{i}\right)}\left(K_{i}(\mathfrak{n}), \phi_{i}\right) \leqslant \sum_{\pi_{\infty} \in \Theta_{i, \infty}} m\left(\pi_{\infty}, \mathfrak{n}\right)
$$


where $m\left(\pi_{\infty}, \mathfrak{n}\right)$ denotes the multiplicity of $\pi_{\infty}$ in $L_{\text {disc }}^{2}\left(U\left(m_{i}, F\right) \backslash U\left(m_{i}, \mathbb{A}\right)\right.$ $\left./ K_{i}(\mathfrak{n})\right)$. In fact it follows from the known cases of the Ramanujan conjecture that $\pi_{\infty}$ is tempered, so $\pi_{\infty}$ appears only in the cuspidal spectrum. Then a theorem of Savin [29] gives $m\left(\pi_{\infty}, \mathfrak{n}\right) \ll N \mathfrak{n}^{m_{i}^{2}}$ for all $\pi_{\infty}$. Combining this with (3) and (4) gives a bound

$$
h_{(2)}^{d}(X(\mathfrak{n}))_{\mathcal{S}} \ll N \mathfrak{n}^{\operatorname{dim} \mathrm{GL}_{N} / U_{\mathcal{S}}+\epsilon}
$$

where $U_{\mathcal{S}}$ is the unipotent radical of $P_{\mathcal{S}}$. Showing that $\operatorname{dim} \mathrm{GL}_{N} / U_{\mathcal{S}} \leqslant N d+1$ completes the proof.

The role played by the cohomological degree in this argument is that $\operatorname{dim} U_{\mathcal{S}}$ must be large if $d$ is small, because of the bound $n_{1} \geqslant N-d$. However, it should be noted that the bound $\operatorname{dim} \mathrm{GL}_{N} / U_{\mathcal{S}} \leqslant N d+1$ does not need to hold if $m_{1} \leqslant 3$, and in these cases there are some additional steps one must take to optimize the argument to obtain the exponent $N d+1$. We will describe them in the course of the proof in the main body except for the following key input, which may be of independent interest. Namely we give in Lemma A.1 a uniform bound (which is significantly better than a trivial bound; see the remark below Lemma A.1) on the dimension of invariant vectors in supercuspidal representations of GL $(r)$ under principal congruence subgroups. By a uniform bound we mean a bound which is independent of the representation (and only depends on the residue field cardinality and the level of congruence subgroup). The asymptotic growth of the invariant dimension is fairly well understood if a representation is fixed but not otherwise. Analogous uniform bounds, on which our paper sheds some light, should be useful for bounding the growth of cohomology of other locally symmetric spaces.

In an earlier version of this paper, we obtained bounds for invariant vectors in supercuspidal representations of $\mathrm{GL}_{2}$ and $\mathrm{GL}_{3}$ (which are the only cases we need here) by a different method, which involved explicitly constructing the representations. The argument for $\mathrm{GL}_{3}$ may be of independent interest, and we have included it in Appendix B. The argument for $\mathrm{GL}_{2}$ is more routine, and may be found in an earlier version of this paper on the arXiv. It applied the construction described in [14, Section 7.A], which for a $p$-adic field $L$ with $p \neq 2$, produces supercuspidals $\pi$ for $\mathrm{GL}_{2}(L)$ from a quadratic extension $L^{\prime} / L$ and a character $\chi$ of $L^{\prime \times}$. Moreover, all supercuspidals are obtained in this way. The construction realizes $\pi$ on the Schwarz space $\mathcal{S}\left(L^{\prime}\right)$, and the formulas it provides for the action of $\mathrm{GL}_{2}(L)$ on $\mathcal{S}\left(L^{\prime}\right)$ easily let one bound the fixed vectors in $\pi$. The bound we obtain is $\operatorname{dim} \pi^{K(n)} \leqslant q^{n}(1+1 / q)$, where $q$ is the order of the residue field of $L$ and $K(n)<\mathrm{GL}_{2}(L)$ is the principal congruence subgroup of depth $n$.

The proof of Theorem 1.2 in fact shows that $h_{(2)}^{d}(X(\mathfrak{n}))_{\mathcal{S}} \ll_{\epsilon} N \mathfrak{n}^{N d+\epsilon}$, except when $\mathcal{S}=(N-d, 1),(1, d)$, or in the exceptional case when $N=4, d=2$, and 
$\mathcal{S}=(2,2)$. Moreover, when $\mathcal{S}=(N-d, 1),(1, d)$ we expect that the bound $h_{(2)}^{d}(X(\mathfrak{n}))_{\mathcal{S}} \ll_{\epsilon} N \mathfrak{n}^{N d+1+\epsilon}$ is sharp when $G$ arises from a Hermitian form, so that the majority of $H_{(2)}^{d}(X(\mathfrak{n}))$ comes from parameters of this shape. By [3, Theorem 10.1], these forms are theta lifted from a Hermitian space of dimension $d$, and it may therefore be possible to prove that Theorem 1.2 is sharp using the theta lift. Note that this is done in [12], but in a slightly different setting to Theorem 1.2. In particular, it is proved there that $h_{(2)}^{d}(X(\mathfrak{n})) \gg N \mathfrak{n}^{N d+1}$ if $G$ arises from a Hermitian form, $d<N / 2$, and $\mathfrak{n}$ has the form $\mathfrak{c p}^{k}$, where $\mathfrak{c}$ is a fixed ideal of $F$ that is sufficiently divisible, and $\mathfrak{p}$ is a fixed prime of $F$ that is inert in $E$.

The reader may be curious as to how we can expect our bound to be sharp, when at a key point in the proof (Lemma 5.2) we seem to bound the dimension of the space of $K$-invariants in a quotient of an induced representation by the invariants in the whole induced representation. We remark that Lemma 5.2 is actually more efficient than this for certain $\mathcal{S}$. In particular, when $\mathcal{S}=(N-d, 1),(1, d)$ (which should give the main contribution), the bound of Lemma 5.2 is sharp.

Finally we remark that it should be possible to adapt much of our arguments to unitary groups of other signatures, notwithstanding combinatorial complexity. However the bound is not going to be optimal as the case of $U(2,2)$ already shows [19]. We would need a sharper uniform bound than $\operatorname{dim} \pi^{K(n)} \ll q^{d(d-1) n / 2}$ when $\pi$ runs over nongeneric representations of $\mathrm{GL}_{d}$.

\section{Notation}

Our notation and discussion in this section are based on [25] and [16]. (Similar summaries are given in [19] and [20] with more details in the quasisplit case.)

Let $N$ be a positive integer. Write $\operatorname{GL}(N)$ for the general linear group. Let $F$ be a field of characteristic zero. Given a quadratic algebra $E$ over $F$, we define $U(N)=U_{E / F}(N)$ to be the quasisplit unitary group in $N$ variables, defined by an antidiagonal matrix $J_{N}$ with $(-1)^{i-1}$ in the $(i, N+1-i)$ entry, as in [16, 0.2.2]. The compact special unitary group in two variables is denoted by SU(2). Let $v(n)$ denote its $n$-dimensional irreducible representation (unique up to isomorphism).

Assume that $F$ is a local or global field of characteristic zero. Write $W_{F}$ for the Weil group of $F$. For any connected reductive group $G$ over $F$, its Langlands dual group is denoted by $\widehat{G}$. Let ${ }^{L} G=\widehat{G} \rtimes W_{F}$ denote the (Weil form of) $L$-group of $G$. Note that ${ }^{L} \mathrm{GL}(N)=\mathrm{GL}(N, \mathbb{C}) \times W_{F}$ and that ${ }^{L} U_{E / F}(N)$ may be explicitly described, cf. [16, 0.2.2].

Now assume that $F$ is local. Define the local Langlands group $L_{F}:=W_{F}$ if $F$ is archimedean and $L_{F}:=W_{F} \times \mathrm{SU}(2)$ otherwise. An $A$-parameter is a continuous homomorphism $\psi: L_{F} \times \operatorname{SL}(2, \mathbb{C}) \rightarrow{ }^{L} G$ commuting with the projection maps onto $W_{F}$ such that $\psi\left(L_{F}\right)$ has relatively compact image in $\widehat{G}$ and that $\psi$ 
restricted to $\operatorname{SL}(2, \mathbb{C})$ is a map of $\mathbb{C}$-algebraic groups into $\widehat{G}$. Two parameters are considered isomorphic if they are conjugate under $\widehat{G}$. Write $\Psi(G)$ or $\Psi(G, F)$ for the set of isomorphism classes of $A$-parameters. Define $\Psi^{+}(G)$ analogously without the condition on relatively compact image. Define $s_{\psi}:=\psi(1,-1)$ for any $\psi \in \Psi^{+}(G)$.

An $L$-parameter is $\psi^{+} \in \Psi^{+}(G)$ which is trivial on the $\operatorname{SL}(2, \mathbb{C})$-factor (external to $L_{F}$ ). The subset of $L$-parameters (up to isomorphism) is denoted by $\Phi(G)$. Any $\psi \in \Psi^{+}(G)$ gives rise to an $L$-parameter $\phi_{\psi}$ by pulling back via the $\operatorname{map} L_{F} \rightarrow L_{F} \times \operatorname{SL}(2, \mathbb{C}), w \mapsto\left(w,\left(\begin{array}{cc}|w|^{1 / 2} & 0 \\ 0 & |w|^{-1 / 2}\end{array}\right)\right)$.

When $G=\operatorname{GL}(N)$, we associate representations $\pi_{\psi}$ and $\rho_{\psi}$ of $\operatorname{GL}(N, F)$ to $\psi \in \Psi^{+}(G)$ by the following recipe from [16, 1.2.2]. We may decompose $\psi=\bigoplus_{i=1}^{k} \psi_{i}$ with $\psi_{i}=\phi_{i} \otimes v\left(n_{i}\right)$ such that $\phi_{i}: L_{F} \rightarrow{ }^{L} \operatorname{GL}\left(m_{i}\right)$ is an irreducible $m_{i}$-dimensional representation of $L_{F}$ and $\sum_{i=1}^{k} m_{i} n_{i}=N$. The local Langlands correspondence associates an irreducible essentially square-integrable representation $\pi_{\phi_{i}}$ of $\operatorname{GL}\left(m_{i}, F\right)$ to $\phi_{i}$. Let $|\operatorname{det}(m)|$ denote the composition of the absolute value on $F^{\times}$with the determinant map on $\operatorname{GL}(m, F)$. Then consider the multiset of representations

$$
\left\{\pi_{\phi_{i}}\left|\operatorname{det}\left(m_{i}\right)\right|^{\left(n_{i}-1\right) / 2}, \pi_{\phi_{i}}\left|\operatorname{det}\left(m_{i}\right)\right|^{\left(n_{i}-3\right) / 2}, \ldots, \pi_{\phi_{i}}\left|\operatorname{det}\left(m_{i}\right)\right|^{\left(1-n_{i}\right) / 2}\right\}_{i=1}^{k} .
$$

This defines a representation of $\prod_{i=1}^{k} \operatorname{GL}\left(m_{i}\right)^{n_{i}}$ viewed as a block diagonal Levi subgroup of $\operatorname{GL}(N)$. Let $\rho_{\psi}$ denote the parabolically induced representation. (The choice of parabolic subgroup does not affect our argument; we will choose the upper triangular one.) The Langlands quotient construction singles out an irreducible subquotient $\pi_{\psi_{i}}$ of the representation of $\operatorname{GL}\left(m_{i} n_{i}, F\right)$ induced from (5) on the Levi subgroup $\operatorname{GL}\left(m_{i}, F\right)^{n_{i}}$. We write $\pi_{\psi}$ for the representation of $\mathrm{GL}(N)$ parabolically induced from $\bigotimes_{i=1}^{k} \pi_{\psi_{i}}$. (In the case of interest, one actually knows that $\pi_{\psi_{i}}$ is unitary by Lemma 6.1 . Thus $\pi_{\psi}$ is the irreducible representation corresponding to $\psi$ by the $A$-packet parametrization.)

As in the introduction, from here throughout the paper, we fix a totally real field $F$ and a totally complex quadratic extension $E$ over $F$ with complex conjugation $c$ in $\operatorname{Gal}(E / F)$. The ring of adeles over $F$ (respectively $E$ ) is denoted by $\mathbb{A}$ (respectively $\mathbb{A}_{E}$ ). We often write $G^{*}$ for $U(N)$ and $G(N)$ for $\operatorname{Res}_{E / F} \operatorname{GL}(N)$. The group $G(N)$ is equipped with involution $\theta: g \mapsto J_{N}{ }^{t} c(g)^{-1} J_{N}^{-1}$, giving rise to the twisted group $\widetilde{G}^{+}(N)=G(N) \rtimes\{1, \theta\}$. Write $\widetilde{G}(N)$ for the coset $G(N) \rtimes \theta$. Let $v$ be a place of $F$. Given any algebraic group $H$ over $F$, we often write $H_{v}$ for $H\left(F_{v}\right)$ or $H \otimes_{F} F_{v}$ (the context will make it clear which one we mean).

For $n \in \mathbb{Z}_{\geqslant 1}$, define $\widetilde{\Phi}_{\text {sim }}(n)$ (a shorthand for $\Phi_{\text {sim }}(\widetilde{G}(n))$ ) to be the set of conjugate self-dual cuspidal automorphic representations of $\operatorname{GL}\left(n, \mathbb{A}_{E}\right)$. Here a representation $\pi$ is considered conjugate self-dual if $\pi \circ c$ is isomorphic to the 
contragredient of $\pi$, or equivalently if $\pi \circ \theta$ is isomorphic to $\pi$. Fix two Hecke characters $\chi_{\kappa}: \mathbb{A}_{E}^{\times} / E^{\times} \rightarrow \mathbb{C}^{\times}$with $\kappa \in\{ \pm 1\}$ as follows: $\chi_{+}$is the trivial character while $\chi_{-}$is an extension of the quadratic character of $\mathbb{A}^{\times} / F^{\times}$associated to $E / F$ by class field theory. We use $\chi_{\kappa}$ to define two base-change $L$-morphisms

$$
\eta_{\chi_{\kappa}}:{ }^{L} U(N) \rightarrow{ }^{L} G(N), \quad \kappa \in\{ \pm 1\},
$$

as follows. Choose $w_{c} \in W_{F} \backslash W_{E}$ so that $W_{F}=W_{E} \coprod W_{E} w_{c}$. Under the identification $\widehat{U}(N)=\operatorname{GL}(N, \mathbb{C})$ and $\widehat{G}(N)=\operatorname{GL}(N, \mathbb{C}) \times \operatorname{GL}(N, \mathbb{C})$, we have (where scalars stand for scalar $N \times N$-matrices whenever appropriate)

$$
\begin{aligned}
\eta_{\chi_{\kappa}}(g \rtimes 1) & =\left(g, J_{N}{ }^{t} g^{-1} J_{N}^{-1}\right) \rtimes 1, \\
\eta_{\chi_{\kappa}}(1 \rtimes w) & =\left(\chi_{\kappa}(w), \chi_{\kappa}^{-1}(w)\right) \rtimes w, \quad w \in W_{E}, \\
\eta_{\chi_{\kappa}}\left(1 \rtimes w_{c}\right) & =(1, \kappa) \rtimes w_{c} .
\end{aligned}
$$

Let us define $\widetilde{\Psi}_{\text {ell }}(N)$, the set of (formal) elliptic parameters for $\widetilde{G}(N)$. Such a parameter is represented by a formal sum $\psi=\boxplus_{i=1}^{k} \psi_{i}$ with $\psi_{i}=\mu_{i} \otimes v\left(n_{i}\right)$ such that the pairs $\left(\mu_{i}, n_{i}\right)$ are mutually distinct, where $\mu_{i} \in \widetilde{\Phi}_{\text {sim }}\left(m_{i}\right), \sum_{i=1}^{k} m_{i} n_{i}=N$. (Two formal sums are identified under permutation of indices.)

Mok defines the sets $\Psi_{2}\left(U(N), \eta_{\chi_{\kappa}}\right.$ ) for $\kappa \in\{ \pm 1\}$. (In [25], he writes $\xi_{\chi_{\kappa}}$ for $\eta_{\chi_{\kappa}}$.) They are identified (via the map $\left(\psi^{N}, \widetilde{\psi}\right) \mapsto \psi^{N}$ of [25, Section 2.4]) with disjoint subsets of $\widetilde{\Psi}_{\text {ell }}(N)$, corresponding to the two ways $U(N)$ can be viewed as a twisted endoscopic group of $\widetilde{G}(N)$ via $\eta_{\chi_{\kappa}}$, characterized by a sign condition. We do not need to recall the sign condition here. It suffices to know that each $\psi \in \Psi_{2}\left(U(N), \eta_{\chi_{\kappa}}\right)$ admits localizations to $\Psi^{+}\left(U(N)_{v}\right)$; see below. We write $\psi^{N}$ for $\psi$ when $\psi$ is viewed as a member of $\widetilde{\Psi}_{\text {ell }}(N)$.

A parameter $\psi$ in $\Psi_{2}\left(U(N), \eta_{\chi_{k}}\right)$ is said to be generic if $n_{i}=1$ for all $1 \leqslant i \leqslant k$ and simple if $k=1$. Write $\Phi_{\text {sim }}\left(U(N), \eta_{\chi_{\kappa}}\right)$ for the subset of simple generic parameters. Theorem 2.4.2 of [25] shows that $\widetilde{\Phi}_{\text {sim }}(N)$ is partitioned into $\Phi_{\text {sim }}\left(U(N), \eta_{\chi_{\kappa}}\right), \kappa \in\{ \pm 1\}$.

To a parameter $\psi \in \Psi_{2}\left(U(N), \eta_{\chi_{\kappa}}\right)$ is associated localizations $\psi_{v} \in \Psi^{+}\left(G_{v}^{*}\right)$ such that $\psi_{v}$ is carried to $\bigoplus_{i=1}^{k} \phi_{\mu_{i, v}} \otimes v\left(n_{i}\right)$ via the $L$-morphism $\eta_{\chi_{\kappa}}:{ }^{L} U(N) \rightarrow$ ${ }^{L} G(N)$, where $\phi_{\mu_{i, v}}$ is the $L$-parameter for $\mu_{i, v}$ (via local Langlands for $\operatorname{GL}\left(m_{i}\right)$ ). For each place $v$ of $F$ split in $E$, fix a place $w$ of $E$ above $v$. Then we have an isomorphism $G_{v}^{*} \simeq \mathrm{GL}\left(N, E_{w}\right)$.

At every finite place $v$ of $F$ where $G_{v}^{*}$ is unramified, fix hyperspecial subgroups $\widetilde{K}_{v}=\operatorname{GL}\left(\mathcal{O}_{F_{v}} \otimes_{\mathcal{O}_{F}} \mathcal{O}_{E}\right)$ of $G\left(N, F_{v}\right)$ and $K_{v}^{*}$ of $G^{*}\left(F_{v}\right)$ (such that they come from global integral models away from finitely many $v$ ). When $v$ is split as $w$ and $c(w)$ in $E$ we have a decomposition $\widetilde{K}_{v}=\widetilde{K}_{w} \times \widetilde{K}_{c(w)}$, and we may identify $K_{v}^{*}$ with $\widetilde{K}_{w}$ via $G_{v}^{*} \simeq \operatorname{GL}\left(N, E_{w}\right)$. 
Finally let $G$ be an inner form of $G^{*}$ over $F$. It can always be promoted to an extended pure inner twist $(\xi, z): G^{*} \rightarrow G,[16,0.3 .3]$. Let $S$ be a set of places of $F$ such that both $G_{v}$ and $G_{v}^{*}$ are unramified for every $v \notin S$. Then fix an isomorphism $G_{v}^{*} \simeq G_{v}$, which is $G(\bar{F})$-conjugate to $(\xi, z)$. We have a hyperspecial subgroup $K_{v} \subset G_{v}$ by transferring $K_{v}^{*}$. So if $v \notin S$ is split in $E$ then $K_{v}$ and $K_{v}^{*}$ are identified with $\operatorname{GL}\left(N, \mathcal{O}_{E, w}\right)$ under the isomorphisms $G_{v} \simeq G_{v}^{*} \simeq$ $\operatorname{GL}\left(N, E_{w}\right)$.

Let $\psi_{v} \in \Psi\left(U(N)_{v}\right)$ for a place $v$ of $F$. This gives rise to a distribution $f \mapsto f\left(\psi_{v}\right)$ on the space of smooth compactly supported functions on $U(N)_{v}$ [25, Theorem 3.2.1].

Given a connected reductive group $H$ over $F_{v}$, a smooth compactly supported function $f$ on $H\left(F_{v}\right)$, and an admissible representation $\pi$ of $H\left(F_{v}\right)$, we write $\operatorname{tr}(\pi(f))$ or $f(\pi)$ for the trace value. Occasionally we also consider a twisted variant when $\tilde{\pi}$ is an admissible representation of $G^{+}\left(N, F_{v}\right)$ and $\tilde{f}$ is a smooth compactly supported function on $G\left(N, F_{v}\right) \rtimes \theta$. Then $\operatorname{tr}(\tilde{\pi}(f))$ will denote the (twisted) trace.

\section{Cohomological representations of $U(N-1,1)$}

In this section, we recall some facts about the cohomological representations of the real Lie group $U(N-1,1)$, which will imply that any global Arthur parameter that contributes to $h_{(2)}^{d}(X(\mathfrak{n}))$ must have a factor $\mu \otimes v(n)$ with $n \geqslant N-d$ by applying results of Bergeron, Millson, and Moeglin. Let $\mathfrak{g}_{0}$ be the real Lie algebra of $U(N-1,1)$, and $K$ a maximal compact subgroup. Write $\mathfrak{g}$ for the complexification of $\mathfrak{g}_{0}$. Similarly the complexification of real Lie algebras $\mathfrak{k}_{0}, \mathfrak{p}_{0}$, and so on will be denoted by $\mathfrak{k}, \mathfrak{p}$, and so on below. The facts we shall need on the cohomological representations of $U(N-1,1)$ are summarized in the following proposition; recall that $p(\pi)$ is the infimum over $p$ for which the $K$-finite matrix coefficients of $\pi$ lie in $L^{p}(G)$.

Proposition 3.1. Let $a, b$ be a pair of integers with $a, b \geqslant 0$ and $a+b \leqslant$ $N-1$, and let $d=a+b$. There is an irreducible unitary representation $\pi_{a, b}$ of $U(N-1,1)$ with the following properties.

(i) We have

$$
\begin{aligned}
& H^{p, q}\left(\mathfrak{g}, K ; \pi_{a, b}\right) \\
& \quad= \begin{cases}\mathbb{C} & \text { if }(p, q)=(a, b)+(k, k), \quad 0 \leqslant k \leqslant N-1-a-b, \\
0 & \text { otherwise. }\end{cases}
\end{aligned}
$$


(ii) Suppose that $d \leqslant N-2$. If $\varphi: \mathbb{C}^{\times} \rightarrow \operatorname{GL}(N, \mathbb{C})$ is the restriction of the Langlands parameter of $\pi_{a, b}$ to $\mathbb{C}^{\times}$, then we have

$$
\varphi(z)=(z / \bar{z})^{(b-a) / 2}|z|^{N-d-1} \oplus(z / \bar{z})^{(b-a) / 2}|z|^{-N+d+1}
$$

(iii) We have $p\left(\pi_{a, b}\right)=2(N-1) / d$.

Moreover, the $\pi_{a, b}$ are the only irreducible unitary representations of $U(N-1,1)$ with $H^{*}(\mathfrak{g}, K ; \pi) \neq 0$.

3.1. The classification of Vogan and Zuckerman. We let $G=U(N-1,1)$, and realize $G$ as the subgroup of $\operatorname{GL}(N, \mathbb{C})$ preserving the Hermitian form $\left|z_{1}\right|^{2}+$ $\cdots+\left|z_{N-1}\right|^{2}-\left|z_{N}\right|^{2}$. The Lie algebra $\mathfrak{g}_{0}$ of $G$ is

$$
\mathfrak{g}_{0}=\left\{A \in M_{N}(\mathbb{C}):{ }^{t} \bar{A}=-I_{N-1,1} A I_{N-1,1}\right\}
$$

where

$$
I_{N-1,1}=\left(\begin{array}{ll}
I_{N-1} & \\
& -1
\end{array}\right) .
$$

The algebras $\mathfrak{k}_{0}$ and $\mathfrak{p}_{0}$ in the Cartan decomposition $\mathfrak{g}_{0}=\mathfrak{k}_{0} \oplus \mathfrak{p}_{0}$ are

$$
\mathfrak{k}_{0}=\left\{\left(\begin{array}{cc}
A & 0 \\
0 & i \theta
\end{array}\right):{ }^{t} \bar{A}=-A, \theta \in \mathbb{R}\right\}, \quad \mathfrak{p}_{0}=\left\{\left(\begin{array}{cc}
0 & z \\
t \bar{z} & 0
\end{array}\right): z \in M_{N-1,1}(\mathbb{C})\right\} .
$$

Let $\mathfrak{t}_{0}$ denote the Cartan subalgebra of $\mathfrak{k}_{0}$ consisting of diagonal matrices. The adjoint action of $K$ on $\mathfrak{p}_{0}$ preserves the natural complex structure, and so we have a decomposition $\mathfrak{p}=\mathfrak{p}_{+} \oplus \mathfrak{p}_{-}$of $K$-modules. We may naturally identify $\mathfrak{g}$ with $M_{n}(\mathbb{C})$, and under this identification we have

$$
\mathfrak{p}_{+}=\left\{\left(\begin{array}{ll}
0 & z \\
0 & 0
\end{array}\right): z \in M_{N-1,1}(\mathbb{C})\right\}, \quad \mathfrak{p}_{-}=\left\{\left(\begin{array}{ll}
0 & 0 \\
z & 0
\end{array}\right): z \in M_{1, N-1}(\mathbb{C})\right\} .
$$

If $\tau_{d}$ is the representation of $K$ on $\bigwedge^{d} \mathfrak{p}$, it is well known [6, VI 4.8-9] that there is a decomposition

$$
\tau_{d}=\bigoplus_{a+b=d} \tau_{a, b}
$$


where $\tau_{a, b}$ is the representation of $K$ on $\bigwedge^{a} \mathfrak{p}_{-} \otimes \bigwedge^{b} \mathfrak{p}_{+}$. Moreover, we have

$$
\tau_{a, b}=\bigoplus_{k=0}^{\min (a, b)} \tau_{a-k, b-k}^{\prime}
$$

for $a+b \leqslant N-1$, where the representations $\tau_{a, b}^{\prime}$ are irreducible with highest weight

$$
\sum_{i=1}^{b} \varepsilon_{i}-\sum_{i=N-a}^{N-1} \varepsilon_{i}+(a-b) \varepsilon_{N} .
$$

Here, $\left\{\varepsilon_{i}\right\}$ is the standard basis for $\mathfrak{t}^{*}$ consisting of elements that are real on $i \mathfrak{t}_{0}$. These decompositions correspond to the Hodge-Lefschetz decomposition for the cohomology of $X(\mathfrak{n})$.

We now recall the classification of cohomological representations of $G$ due to Vogan and Zuckerman [31]. We choose an element $H \in i \mathfrak{t}_{0}$, so that $\operatorname{ad}(H)$ has real eigenvalues. We let $\mathfrak{q} \subset \mathfrak{g}$ be the parabolic subalgebra $\mathfrak{l}+\mathfrak{u}$, where $\mathfrak{l}=Z_{\mathfrak{g}}(H)$ and $\mathfrak{u}$ is the sum of all the eigenspaces for $\operatorname{ad}(H)$ with positive eigenvalues. Because $\mathfrak{k}$ and $\mathfrak{p}_{ \pm}$are stable under $\operatorname{ad}(H)$, we have $\mathfrak{u}=\mathfrak{u} \cap \mathfrak{k}+\mathfrak{u} \cap \mathfrak{p}_{-}+\mathfrak{u} \cap \mathfrak{p}_{+}$. We define $R_{ \pm}=\operatorname{dim}\left(\mathfrak{u} \cap \mathfrak{p}_{ \pm}\right)$and $R=R_{+}+R_{-}$, and let $\mu=2 \rho(\mathfrak{u} \cap \mathfrak{p})$, which is the sum of the roots of $\mathfrak{t}$ in $\mathfrak{u} \cap \mathfrak{p}$. We fix a set of positive roots for $\mathfrak{t}$ in $\mathfrak{l} \cap \mathfrak{k}$ so that a positive root system for $\mathfrak{t}$ in $\mathfrak{k}$ is determined (together with $\mathfrak{u} \cap \mathfrak{k}$ ). Then $\mu$ is a highest weight for the positive root system.

The main theorem of Vogan and Zuckerman is that there is a unique irreducible unitary representation $A_{\mathfrak{q}}$ of $G$ with the following properties:

- $A_{\mathfrak{q}}$ has the same infinitesimal character as the trivial representation.

- $A_{\mathfrak{q}}$ contains the $K$-type with highest weight $\mu$.

(Note that the general unitarity of the representations $A_{\mathfrak{q}}$ is proved in [30].) They also show that any irreducible unitary representation of $G$ with nonzero ( $\mathfrak{g}$, $K$ )-cohomology (with trivial coefficients) must be of the form $A_{\mathfrak{q}}$ for some q. It is clear that $A_{\mathfrak{q}}$ only depends on $\mathfrak{u} \cap \mathfrak{p}$. Moreover, we have [31, Proposition 6.19]

$$
H^{R_{+}+p, R_{-}+p}\left(\mathfrak{g}, K ; A_{\mathfrak{q}}\right) \simeq \operatorname{Hom}_{\mathfrak{l n k}}\left(\wedge^{2 p}(\mathfrak{l} \cap \mathfrak{p}), \mathbb{C}\right), \quad p \geqslant 0,
$$

and

$$
H^{p, q}\left(\mathfrak{g}, K ; A_{\mathfrak{q}}\right)=0
$$

for other $(p, q)$, that is if $p-q \neq R_{+}-R_{-}$. 
Write $H_{1}, \ldots, H_{N}$ for the entries of the real diagonal matrix $H$. Because $A_{\mathfrak{q}}$ only depends on the orbit of $H$ under the Weyl group of $K$, we may assume that $H_{1} \geqslant \cdots \geqslant H_{N-1}$. The subspace $\mathfrak{u} \cap \mathfrak{p}$, and hence $A_{\mathfrak{q}}$, only depends on the number of $H_{i}-H_{N}$ that are positive, negative, and zero. Therefore, if $a$ and $b$ are the number of $H_{i}-H_{N}$ that are positive and negative, respectively, then we have $H_{a+1}=\cdots=H_{N-1-b}=H_{N}$, while we may assume that all the remaining $H_{i}$ are distinct. It may be seen that $R_{+}=a$ and $R_{-}=b$, and

$$
\mu=\sum_{i=1}^{a} \varepsilon_{i}-\sum_{i=N-b}^{N-1} \varepsilon_{i}-(a-b) \varepsilon_{N} .
$$

The representation $A_{\mathfrak{q}}$ depends only on $a$ and $b$, and we denote it by $\pi_{a, b}$.

To prove (iii), we will need the description of $\pi_{a, b}$ as a Langlands quotient when $a+b<N-1$, which is given by Vogan and Zuckerman in [31, Theorem 6.16]. Define

$$
V=\left(\begin{array}{cc} 
& \\
& 0_{N-2} \\
1 &
\end{array}\right)
$$

and let $\mathfrak{a}_{0}=\mathbb{R} V$ so that $\mathfrak{a}_{0}$ is a maximal abelian subalgebra of $\mathfrak{p}_{0}$. Let $A=\exp \left(\mathfrak{a}_{0}\right)$ be the corresponding subgroup. Define $\alpha \in \mathfrak{a}^{*}$ by $\alpha(V)=1$. The roots of $\mathfrak{a}$ in $\mathfrak{g}$ are $\pm \alpha$ and $\pm 2 \alpha$ with multiplicities $2(N-2)$ and 1 , respectively, so that $\rho=(N-1) \alpha$. Let $U$ be the unipotent subgroup corresponding to the positive roots. Let $M=Z_{K}(V)$, so that

$$
M=\left\{\left(\begin{array}{ccc}
e^{i \theta} & & \\
& X & \\
& & e^{i \theta}
\end{array}\right): X \in U(N-2), \theta \in \mathbb{R}\right\} .
$$

Let $\mathfrak{t}_{M} \subset \mathfrak{t}$ be the diagonal Cartan subalgebra in $\mathfrak{m}$. Let $\sigma$ be the irreducible representation of $M$ with highest weight given by the restriction to $\mathfrak{t}_{M}$ of

$$
\sum_{i=2}^{a+1} \varepsilon_{i}-\sum_{i=N-b}^{N-1} \varepsilon_{i}+(b-a) \varepsilon_{1}
$$

Let $v=(N-1-d) \alpha$. We define $I_{v, \sigma}$ to be the unitarily normalized induction from $P=M A U$ to $G$ of the representation $\sigma \otimes e^{v} \otimes 1$. Then $\pi_{a, b}$ is the Langlands quotient of $I_{v, \sigma}$.

3.2. Proof of Proposition 3.1. The assertion that $\pi_{a, b}$ are the only representations with nonzero cohomology is clear, because any such representation is 
isomorphic to $A_{\mathfrak{q}}$ for some $\mathfrak{q}$. The calculation of $H^{p, q}\left(\mathfrak{g}, K ; \pi_{a, b}\right)$ in condition (i) follows from (9) and (10) after we compute $\operatorname{Hom}_{\mathfrak{l} \cap \mathfrak{k}}\left(\wedge^{2 p}(\mathfrak{l} \cap \mathfrak{p}), \mathbb{C}\right)$. Our assumption on $H$ implies that $\mathfrak{l}_{0} \simeq \mathfrak{u}(N-d-1,1) \times \mathfrak{u}(1)^{d}$, and $\mathfrak{l}=\mathfrak{l} \cap \mathfrak{k} \oplus \mathfrak{l} \cap \mathfrak{p}$ is the standard Cartan decomposition of $\mathfrak{l}$. We wish to show that the trivial representation of $\mathfrak{l} \cap \mathfrak{k}$ occurs exactly once in $\wedge^{2 p}(\mathfrak{l} \cap \mathfrak{p})$ for all $0 \leqslant p \leqslant N-d-1$, but this follows from the decompositions (6) and (7) for $\mathfrak{u}(N-d-1,1)$, combined with the fact that $\tau_{p, q}^{\prime}$ is trivial if and only if $p=q=0$ as one sees from the highest weight formula (8).

The description of the Langlands parameter of $\pi_{a, b}$ in (ii) follows from [2, Section 5.3].

To prove assertion (iii), we may assume that $a+b<N-1$ as otherwise $\pi_{a, b}$ lies in the discrete series. When $a+b<N-1$, the assertion follows from our description of $\pi_{a, b}$ as a Langlands quotient, and well-known asymptotics for matrix coefficients, which we recall from Knapp [17]. Let $\bar{P}=M A \bar{U}$ be the opposite parabolic to $P$, and let $\bar{I}_{\sigma, v}$ be the normalized induction of $\sigma \otimes e^{v} \otimes 1$ from $\bar{P}$ to $G$. Let $A(\sigma, v): I_{\sigma, v} \rightarrow \bar{I}_{\sigma, v}$ be the intertwiner

$$
A(\sigma, v) f(g)=\int_{\bar{U}} f(u g) d u,
$$

which converges by [17, VII, Proposition 7.8]. Then the image of $A(\sigma, v)$ is isomorphic to the Langlands quotient $\pi_{a, b}$ of $I_{\sigma, v}$. We introduce the pairing on $I_{\sigma, v}$ given by

$$
\langle f, g\rangle=\int_{K}\langle f(k), g(k)\rangle_{\sigma} d k
$$

where $\langle\cdot, \cdot\rangle_{\sigma}$ denotes a choice of inner product on $\sigma$. If we choose $g \in I_{\sigma, v}$ to pair trivially with the kernel of $A(\sigma, v)$, then $\left\langle I_{\sigma, v}(\cdot) f, g\right\rangle$ is a matrix coefficient of $\pi_{a, b}$, and all coefficients are realized in this way. The asymptotic behavior of the coefficients is given by [17, VII, Lemma 7.23], which states that

$$
\lim _{a \rightarrow \infty} e^{(\rho-v) \log a}\left\langle I_{\sigma, v}(a) f, g\right\rangle=\langle A(\sigma, v) f(1), g(1)\rangle_{\sigma} .
$$

As $v=(N-d-1) \alpha$, [17, VIII, Theorem 8.48] implies that $p\left(\pi_{a, b}\right) \leqslant$ $2(N-1) / d$. It also follows from that theorem that to prove $p\left(\pi_{a, b}\right)=2(N-1) / d$, we need only show that the right hand side of (11) is nonzero for some choice of $f$ and $g$, subject to the condition that $g$ pairs trivially with ker $A(\sigma, v)$. To do this, choose $f \in I_{\sigma, v}$ such that $A(\sigma, v) f \neq 0$, and some nonzero $g$ of the required type. Because $A(\sigma, v)$ is an intertwiner, after translating $f$ by $K$ we may assume that $A(\sigma, v) f(1) \neq 0$. Because $\operatorname{ker} A(\sigma, v)$ is an invariant subspace, we may likewise assume that $g(1) \neq 0$. Because $\sigma$ was irreducible, translating by $M$ we may also assume that $\langle A(\sigma, v) f(1), g(1)\rangle_{\sigma} \neq 0$ as required. 


\section{Application of the global classification}

As in the notation section, $(\xi, z): G^{*} \rightarrow G$ is an extended pure inner twist of the quasisplit unitary group $G^{*}=U(N)$ over $F$. We always assume that $G_{v_{0}}$ is isomorphic to $U(N-1,1)$ at a real place $v_{0}$ of $F$ and that $G_{v}$ is compact at all other real places $v$. Although much of our argument works for general inner forms, the assumption significantly simplifies some combinatorial and representation-theoretic arguments (especially of Section 3) and ensures that we obtain expectedly optimal upper bounds in all degrees in the main theorem.

Let $\psi \in \Psi_{2}\left(G^{*}, \eta_{\chi_{\kappa}}\right)$. The main local theorem of [16] defines local packets $\Pi_{\psi_{v}}(G, \xi)$ consisting of finitely many (possibly reducible and nonunitary) representations of $G_{v}$ such that $\Pi_{\psi_{v}}(G, \xi)$ contains an unramified representation (relative to $K_{v}$ ) at all but finitely many $v$. (The issue is that $\psi_{v} \in \Psi^{+}\left(G_{v}^{*}\right)$ is not known to be in $\Psi\left(G_{v}^{*}\right)$ in general although it is expected. However this is actually known for parameters contributing to cohomology from the known cases of the Ramanujan conjecture, see Section 6. It follows that all representations in the local packets we will consider are irreducible and unitary.) The global packet $\Pi_{\psi}(G, \xi)$ consists of restricted tensor products $\pi=\bigotimes_{v}^{\prime} \pi_{v}$ with $\pi_{v} \in \Pi_{\psi_{v}}(G, \xi)$. The parameter $\psi$ determines a sign character $\epsilon_{\psi}$ on a certain centralizer group (in $\widehat{G}$ ) attached to $\psi$, and [16] defines a subset $\Pi_{\psi}\left(G, \xi, \epsilon_{\psi}\right)$ of $\Pi_{\psi}(G, \xi)$ by imposing a sign condition. We need not recall the condition as it will be soon ignored along the way to an upper bound. Theorem 1.7.1 of [16] asserts the following.

THEOREM 4.1. There is a $G(\mathbb{A})$-module isomorphism

$$
L_{\mathrm{disc}}^{2}(G(F) \backslash G(\mathbb{A})) \simeq \bigoplus_{\psi \in \Psi_{2}\left(G^{*}, \eta_{\chi \kappa}\right)} \bigoplus_{\pi \in \Pi_{\psi}\left(G, \xi, \epsilon_{\psi}\right)} \pi
$$

Let $S$ be a finite set of finite places of $F$ containing all places at which $E$ or $G$ ramify. Let $\mathfrak{n} \subset \mathcal{O}_{F}$ be a nonzero ideal whose prime factors are split in $E$ and do not lie in $S$. In Section 2 we have introduced hyperspecial subgroups $K_{v}$ of $G\left(F_{v}\right)$ when $v \notin S$. For $v \in S$ let $K_{v}$ be an arbitrary open compact subgroup of $G\left(F_{v}\right)$. Now we define the congruence subgroup $K(\mathfrak{n})=\prod_{v} K(\mathfrak{n})_{v}$, where $K(\mathfrak{n})_{v}$ is given as follows for each finite place $v$. Define $K(\mathfrak{n})_{v}$ to be $K_{v}$ if $v$ does not divide $\mathfrak{n}$. If $v \mid \mathfrak{n}$ then we have fixed an isomorphism $K_{v} \simeq \operatorname{GL}\left(N, \mathcal{O}_{E_{w}}\right)$, and $K(\mathfrak{n})_{v}$ is the subgroup of $K_{v}$ consisting of elements congruent to the identity modulo $\mathfrak{n}$. Let $K_{\infty}$ denote a maximal compact subgroup of $G\left(F \otimes_{\mathbb{Q}} \mathbb{R}\right)$. Often we write $[G]$ for the quotient $G(F) \backslash G(\mathbb{A})$, and likewise when $G$ is replaced with quasisplit unitary groups. 
We would like to investigate the cohomology of the arithmetic manifold

$$
X(\mathfrak{n})=G(F) \backslash G(\mathbb{A}) / K(\mathfrak{n}) K_{\infty} .
$$

Since $G\left(F \otimes_{\mathbb{Q}} \mathbb{R}\right) / K_{\infty}$ is isomorphic to the symmetric space $U(N-1,1) /$ $(U(N-1) \times U(1))$, which has complex dimension $N-1$, we see that the complex dimension of $X(\mathfrak{n})$ is also $N-1$.

We take the first step in proving Theorem 1.2 on bounding the $L^{2}$-Lefschetz numbers $h_{(2)}^{d}(X(\mathfrak{n}))$ in degrees $0 \leqslant d<N-1$, as $\mathfrak{n}$ varies. Write $h^{d}\left(\mathfrak{g}, K_{\infty} ; \pi_{\infty}\right)$ for $\operatorname{dim} H^{d}\left(\mathfrak{g}, K_{\infty} ; \pi_{\infty}\right)$. Matsushima's formula (see [6] in the noncompact case) gives

$$
h_{(2)}^{d}(X(\mathfrak{n}))=\sum_{\pi \subset L_{\text {disc }}^{2}([G])} m(\pi) h^{d}\left(\mathfrak{g}, K_{\infty} ; \pi_{\infty}\right) \operatorname{dim} \pi_{f}^{K(\mathfrak{n})},
$$

where the sum runs over irreducible $G(\mathbb{A})$-subrepresentations $\pi$ of $L_{\text {disc }}^{2}([G])$ up to isomorphism, and $m(\pi):=\operatorname{dim}_{\operatorname{Hom}_{G(\mathbb{A})}}\left(\pi, L_{\text {disc }}^{2}([G])\right)$ denotes the multiplicity of $\pi$.

Combining this with Theorem 4.1 gives

$$
h_{(2)}^{d}(X(\mathfrak{n})) \leqslant \sum_{\psi \in \Psi_{2}\left(G^{*}, \eta_{\chi_{K}}\right)} \sum_{\pi \in \Pi_{\psi}(G, \xi)} h^{d}\left(\mathfrak{g}, K_{\infty} ; \pi_{\infty}\right) \operatorname{dim} \pi_{f}^{K(\mathfrak{n})} .
$$

\section{Bounding the contribution of a single parameter}

In this section, we bound the contribution of a single parameter $\psi$ to the right hand side of (13). The form of our bound will depend on the shape of $\psi$, and so throughout this section we shall fix a shape $\mathcal{S}=\left(n_{1}, m_{1}\right), \ldots,\left(n_{k}, m_{k}\right)$ and define $\Psi_{2}\left(G^{*}, \eta_{\chi_{\kappa}}\right)_{\mathcal{S}}$ to be the set of parameters with that shape. If $\psi \in \Psi_{2}\left(G^{*}, \eta_{\chi_{\kappa}}\right)_{\mathcal{S}}$, we define $\mu_{i} \in \widetilde{\Phi}_{\text {sim }}\left(m_{i}\right)$ to be such that $\psi^{N}=\boxplus_{i \geqslant 1} \mu_{i} \otimes v\left(n_{i}\right)$. Each $\mu_{i}$ represents a simple generic parameter $\phi_{i} \in \Phi_{\text {sim }}\left(U\left(m_{i}\right), \eta_{\chi_{\kappa_{i}}}\right)$ for a unique sign $\kappa_{i} \in\{ \pm 1\}$ determined as in $[25,(2.4 .8)]$. We define

$$
\begin{aligned}
\tau(\mathcal{S}) & =\left(\begin{array}{c}
N \\
2
\end{array}\right)-\sum_{i \geqslant 1} n_{i}\left(\begin{array}{c}
m_{i} \\
2
\end{array}\right), \\
\tau_{1}(\mathcal{S}) & =\left(\begin{array}{c}
N \\
2
\end{array}\right)-\left(\begin{array}{c}
n_{1} \\
2
\end{array}\right)-\sum_{i \geqslant 2} n_{i}\left(\begin{array}{c}
m_{i} \\
2
\end{array}\right), \\
\tau_{2}(\mathcal{S}) & =\tau(\mathcal{S})+\left(n_{1}-1\right)+\epsilon, \\
\tau_{3}(\mathcal{S}) & =\tau(\mathcal{S})+3\left(n_{1}-1\right)+\epsilon .
\end{aligned}
$$


Here, $\epsilon>0$ is an arbitrarily small constant that may vary from line to line. Any implied constants in bounds for quantities containing $\tau_{2}(\mathcal{S})$ or $\tau_{3}(\mathcal{S})$ will be assumed to depend on $\epsilon$. We also define

$$
\sigma(\mathcal{S})=\sigma_{2}(\mathcal{S})=\sigma_{3}(\mathcal{S})=\sum_{i=1}^{k} n_{i}-1, \quad \sigma_{1}(\mathcal{S})=\sum_{i=2}^{k} n_{i}
$$

For each $1 \leqslant i \leqslant k$ and finite place $v$, define a compact open subgroup $K_{i, v}$ of $U\left(m_{i}\right)_{v}$ as follows. If $v \notin S$, then $K_{i, v}$ is the standard hyperspecial subgroup, and if $v \in S$ then $K_{i, v}$ will be chosen during the proof of Proposition 5.1. Let $K_{i}=\prod_{v} K_{i, v}$, and let $K_{i}(\mathfrak{n})$ be the principal congruence subgroup of $K_{i}$ of level $\mathfrak{n}$. Let $P \subset \mathrm{GL}(N)$ be the standard parabolic subgroup with Levi $\prod_{i=1}^{k} \operatorname{GL}\left(m_{i}\right)^{n_{i}}$.

PROPOSITION 5.1. There is a choice of $K_{i, v}$ for $v \in S$ with the following property. Let $\psi \in \Psi_{2}\left(G^{*}, \eta_{\chi_{\kappa}}\right)_{\mathcal{S}}$, and assume that $\phi_{i}$ (arising from $\psi$ as above) is bounded everywhere for each $1 \leqslant i \leqslant k$. Then

$$
\sum_{\pi \in \Pi_{\psi}(G, \xi)} \operatorname{dim} \pi_{f}^{K(\mathfrak{n})} \ll \prod_{v \mid \mathfrak{n}}\left(1+1 / q_{v}\right)^{\sigma(\mathcal{S})} N \mathfrak{n}^{\tau(\mathcal{S})} \prod_{i \geqslant 1}\left(\sum_{\pi_{i} \in \Pi_{\phi_{i}}\left(U\left(m_{i}\right)\right)} \operatorname{dim} \pi_{i, f}^{K_{i}(\mathfrak{n})}\right)^{n_{i}} .
$$

Moreover, if $m_{1}=l$ with $l=1,2,3$, we have

$$
\begin{aligned}
\sum_{\pi \in \Pi_{\psi}(G, \xi)} \operatorname{dim} \pi_{f}^{K(\mathfrak{n})} \ll & \prod_{v \mid \mathfrak{n}}\left(1+1 / q_{v}\right)^{\sigma_{l}(\mathcal{S})} N \mathfrak{n}^{\tau_{l}(\mathcal{S})} \\
& \sum_{\pi_{1} \in \Pi_{\phi_{1}}\left(U\left(m_{1}\right)\right)} \operatorname{dim} \pi_{1, f}^{K_{1}(\mathfrak{n})} \prod_{i \geqslant 2}\left(\sum_{\pi_{i} \in \Pi_{\phi_{i}}\left(U\left(m_{i}\right)\right)} \operatorname{dim} \pi_{i, f}^{K_{i}(\mathfrak{n})}\right)^{n_{i}} .
\end{aligned}
$$

The first step in proving Proposition 5.1 is to write both sides as a product over the finite places. We describe this in the case of the first inequality, as the second is similar. We have

$$
\sum_{\pi \in \Pi_{\psi}(G, \xi)} \operatorname{dim} \pi_{f}^{K(\mathfrak{n})}=\prod_{v \nmid \infty} \sum_{\pi_{v} \in \Pi_{\psi_{v}}\left(G_{v}, \xi_{v}\right)} \operatorname{dim} \pi_{v}^{K_{v}(\mathfrak{n})}
$$

and

$$
\prod_{i \geqslant 1}\left(\sum_{\pi_{i} \in \Pi_{\phi_{i}}\left(U\left(m_{i}\right)\right)} \operatorname{dim} \pi_{i, f}^{K_{i}(\mathfrak{n})}\right)^{n_{i}}=\prod_{v \nmid \infty} \prod_{i \geqslant 1}\left(\sum_{\pi_{i, v} \in \Pi_{\phi_{i, v}}\left(U\left(m_{i}\right)\right)} \operatorname{dim} \pi_{i, v}^{K_{i, v}(\mathfrak{n})}\right)^{n_{i}} .
$$


It therefore suffices to prove that

$$
\sum_{\pi_{v} \in \Pi_{\psi_{v}}\left(G_{v}, \xi_{v}\right)} \operatorname{dim} \pi_{v}^{K_{v}(\mathfrak{n})} \leqslant C_{v}\left(1+O\left(q_{v}^{-2}\right)\right) \prod_{i \geqslant 1}\left(\sum_{\pi_{i, v} \in \Pi_{\phi_{i, v}}\left(U\left(m_{i}\right)\right)} \operatorname{dim} \pi_{i, v}^{K_{i, v}(\mathfrak{n})}\right)^{n_{i}}
$$

for all finite $v$. Here, the constant $C_{v}$ may be arbitrary for $v \in S$, while for $v \notin S$ it is either 1 if $v$ is inert in $E / F$, or the $v$-component of the constant in (15) if $v$ is split. It is important to keep $C_{v}$ independent of $\psi_{v}$ and $\mathfrak{n}$ (but it could depend on $K_{v}$ ) for the application to the proof of the main theorem. We divide the proof of equation (17) into four cases, depending on whether $v$ is split in $E$, and whether $v \in S$. Thus the proof of Proposition 5.1 will be complete by Lemmas 5.2-5.5.

5.1. A remark on the representations $\rho_{\psi}$. In Section 5, we will need to modify the definition of the local representation $\rho_{\psi}$ given in Section 2 to make it compatible with our global arguments. We first assume that $v$ is split in $E / F$. Let $\psi$ be as in Proposition 5.1, and consider the localization $\psi_{v}$, which is a parameter for $\operatorname{GL}\left(N, E_{w}\right)$. In Section 2, we associated a representation $\rho_{\psi_{v}}$ to $\psi_{v}$, by decomposing $\psi_{v}$ into irreducibles and applying the recipe (5). We may modify this definition, by instead using the decomposition $\psi_{v}=\bigoplus_{i \geqslant 1} \phi_{v, i} \otimes v\left(n_{i}\right)$ with $\phi_{v, i} \in \Phi_{v}\left(m_{i}\right)$ associated to the shape $\mathcal{S}$ (so that the $\phi_{v, i}$ are not necessarily irreducible), and in Section 5 we will let $\rho_{\psi_{v}}$ denote the representation of $\operatorname{GL}(N$, $E_{w}$ ) obtained in this way. In particular, $\rho_{\psi_{v}}$ is induced from a parabolic of $\operatorname{GL}(N)$ with Levi $\prod_{i=1}^{k} \operatorname{GL}\left(m_{i}\right)^{n_{i}}$. If $v$ is not split in $E / F$, then we have a local parameter $\psi_{v}^{N}$ for $\operatorname{GL}\left(N, E_{w}\right)$, and we modify the definition of the induced representation $\rho_{\psi_{v}^{N}}$ in the same way.

If we denote the result of the old construction by $\rho_{\psi_{v}}^{\prime}$, then $\rho_{\psi_{v}}$ and $\rho_{\psi_{v}}^{\prime}$ have the same composition series. This implies that $\pi_{\psi_{v}}$ is a subquotient of $\rho_{\psi_{v}}$, which is all that matters for most of our arguments. The only exception to this is in Lemma 5.2, which will be discussed in the course of the proof.

5.2. $v$ split in $\boldsymbol{E} / \boldsymbol{F}, \boldsymbol{v} \notin S$. These $v$ are the only ones which require us to consider the special cases $m_{1}=1,2,3$ of Proposition 5.1 separately. In this case, the local packets under consideration each contain a single representation of $\operatorname{GL}\left(N, F_{v}\right)$ or $\operatorname{GL}\left(m_{i}, F_{v}\right)$. The bound we prove, Lemma 5.2, is an application of the fact that the representation in $\Pi_{\psi_{v}}\left(G_{v}, \xi_{v}\right)$ is a subquotient of an explicit induced representation.

LEMmA 5.2. Let $\Pi_{\psi_{v}}(G, \xi)=\left\{\pi_{v}\right\}$ and $\Pi_{\phi_{i, v}}\left(U\left(m_{i}\right)\right)=\left\{\pi_{i, v}\right\}$. We have

$$
\operatorname{dim} \pi_{v}^{K(\mathfrak{n})_{v}} \leqslant\left(1+1 / q_{v}\right)^{\sigma(\mathcal{S})}\left(1+O\left(q_{v}^{-2}\right)\right) N \mathfrak{n}_{v}^{\tau(\mathcal{S})} \prod_{i \geqslant 1}\left(\operatorname{dim} \pi_{i, v}^{K_{i}(\mathfrak{n})_{v}}\right)^{n_{i}},
$$


and if $m_{1}=l$ with $l=1,2,3$ we have

$$
\begin{aligned}
\operatorname{dim} \pi_{v}^{K(\mathfrak{n})_{v}} \leqslant & C\left(\epsilon, q_{v}\right)\left(1+1 / q_{v}\right)^{\sigma_{l}(\mathcal{S})}\left(1+O\left(q_{v}^{-2}\right)\right) N \mathfrak{n}_{v}^{\tau_{l}(\mathcal{S})} \operatorname{dim} \pi_{1, v}^{K_{1}(\mathfrak{n})_{v}} \\
& \times \prod_{i \geqslant 2}\left(\operatorname{dim} \pi_{i, v}^{K_{i}(\mathfrak{n})_{v}}\right)^{n_{i}}
\end{aligned}
$$

The terms involving $1+1 / q_{v}$ only need to be included if $v \mid \mathfrak{n}$. The term $C\left(\epsilon, q_{v}\right)=1$ if $l=1$ or $q_{v}$ is greater than a constant depending on $\epsilon$.

Proof. We recall the identification $G_{v}=\operatorname{GL}\left(N, E_{w}\right)$, which carries $K_{v}$ to $\widetilde{K}_{w}$. View $\psi_{v}$ as a member of $\Psi\left(\operatorname{GL}\left(N, E_{w}\right)\right)$. As in Section 5.1, we have an irreducible subquotient $\pi_{v}=\pi_{\psi_{v}}$ of an induced representation $\rho_{w}=\rho_{\psi_{v}}$ of $\operatorname{GL}\left(N, E_{w}\right)$. Let $P_{w}$ denote the block upper triangular parabolic subgroup from which $\rho_{w}$ is induced. (So the Levi factor of $P_{w}$ is $\prod_{i=1}^{k} \operatorname{GL}\left(m_{i}\right)^{n_{i}}$.)

We shall prove the first bound using $\operatorname{dim} \pi_{v}^{K(\mathfrak{n})_{v}} \leqslant \operatorname{dim} \rho_{w}^{\widetilde{K}(\mathfrak{n})_{w}}$. We have

$$
\operatorname{dim} \rho_{w}^{\widetilde{K}(\mathfrak{n})_{w}}=\left[\widetilde{K}_{w}: \widetilde{K}_{w} \cap \widetilde{K}(\mathfrak{n})_{w} P_{w}\right] \prod_{i \geqslant 1}\left(\operatorname{dim} \mu_{i, w}^{\widetilde{K}_{i}(\mathfrak{n})_{w}}\right)^{n_{i}}
$$

The result then follows from the fact that $\left[\widetilde{K}_{w}: \widetilde{K}_{w} \cap \widetilde{K}(\mathfrak{n})_{w} P_{w}\right]=1$ if $v \nmid \mathfrak{n}$, while if $v \mid \mathfrak{n}$ we have

$$
\left[\widetilde{K}_{w}: \widetilde{K}_{w} \cap \widetilde{K}(\mathfrak{n})_{w} P_{w}\right]=\left(1+1 / q_{v}\right)^{\sigma(\mathcal{S})}\left(1+O\left(q_{v}^{-2}\right)\right) N \mathfrak{n}_{v}^{\tau(\mathcal{S})},
$$

and the fact that $\pi_{i, v}$ are isomorphic to $\mu_{i, w}$ so that $\operatorname{dim} \mu_{i, w}^{\widetilde{K}_{i}(\mathfrak{n})_{w}}=\operatorname{dim} \pi_{i, v}^{K_{i}(\mathfrak{n})_{v}}$.

The case $m_{1}=1$ is the only place that we need to know the exact definition of $\pi_{v}$, not just that it is a subquotient of $\rho_{w}$. Let $\rho_{w}^{\prime}$ be the induced representation of $\operatorname{GL}\left(N, E_{w}\right)$ associated to $\psi_{v}$ in Section 2. Because $\phi_{i, v}$ are bounded, $\pi_{v}$ is the unique irreducible quotient of $\rho_{w}^{\prime}$. Because $m_{1}=1, \rho_{w}^{\prime}$ is induced from a standard parabolic $P_{w}^{\prime}$ with Levi factor $L_{w}^{\prime}=\mathrm{GL}(1)^{n_{1}} \times \prod_{j} \mathrm{GL}\left(t_{j}\right)$ for some $t_{j}$. Moreover, the representation one induces is given on the GL(1 $)^{n_{1}}$ factor of $L_{w}^{\prime}$ by

$$
|\operatorname{det}|^{\left(n_{1}-1\right) / 2} \mu_{1, w} \otimes \cdots \otimes|\operatorname{det}|^{\left(-n_{1}+1\right) / 2} \mu_{1, w} .
$$

Let $\left(P_{w}^{1}\right)^{\prime}$ be the parabolic obtained by modifying $P_{w}^{\prime}$ in the upper-left $n_{1} \times n_{1}$ block so that the GL(1 $)^{n_{1}}$ factor in the Levi is replaced by $\operatorname{GL}\left(n_{1}\right)$. Let $\left(\rho_{w}^{1}\right)^{\prime}$ be the representation induced from $\left(P_{w}^{1}\right)^{\prime}$ using the same data as $\rho_{w}^{\prime}$, except that one takes the representation $\mu_{1, w} \circ \operatorname{det}\left(n_{1}\right)$ on the new Levi factor $\operatorname{GL}\left(n_{1}, E_{w}\right)$. As $\left(\rho_{w}^{1}\right)^{\prime}$ is a quotient of $\rho_{w}^{\prime}, \pi_{v}$ is also a quotient of $\left(\rho_{w}^{1}\right)^{\prime}$.

We may perform a similar modification to $\rho_{w}$, to define a representation $\rho_{w}^{1}$ induced from the standard parabolic $P_{w}^{1}$ with Levi GL $\left(n_{1}\right) \times \prod_{i \geqslant 2} \operatorname{GL}\left(m_{i}\right)^{n_{i}}$. As 
$\rho_{w}^{1}$ and $\left(\rho_{w}^{1}\right)^{\prime}$ have the same composition series, $\pi_{v}$ is a subquotient of $\rho_{w}^{1}$. It follows that $\operatorname{dim} \pi_{v}^{K(\mathfrak{n})_{v}} \leqslant \operatorname{dim}\left(\rho_{w}^{1}\right)^{\widetilde{K}(\mathfrak{n})_{w}}$, and

$$
\operatorname{dim}\left(\rho_{w}^{1}\right)^{\widetilde{K}(\mathfrak{n})_{w}}=\left[\widetilde{K}_{w}: \widetilde{K}_{w} \cap \widetilde{K}(\mathfrak{n})_{w} P_{w}^{1}\right] \operatorname{dim} \mu_{1, w}^{\widetilde{K}_{1}(\mathfrak{n})_{w}} \prod_{i \geqslant 2}\left(\operatorname{dim} \mu_{i, w}^{\widetilde{K}_{i}(\mathfrak{n})_{w}}\right)^{n_{i}} .
$$

The result follows as before, after calculating $\left[\widetilde{K}_{w}: \widetilde{K}_{w} \cap \widetilde{K}(\mathfrak{n})_{w} P_{w}^{1}\right]$.

In the cases $m_{1}=2,3$, we bound all but one of the factors of $\operatorname{dim} \pi_{1, v}^{K_{1}(\mathfrak{n})_{v}}$ in (18) using the representation theory of $\mathrm{GL}\left(m_{1}, F_{v}\right)$. In particular, applying Corollary A.4 in (18) gives

$$
\begin{gathered}
\operatorname{dim} \pi_{v}^{K(\mathfrak{n})_{v}} \leqslant C\left(\epsilon, q_{v}\right)\left(1+1 / q_{v}\right)^{\sigma(\mathcal{S})}\left(1+O\left(q_{v}^{-2}\right)\right) N \mathfrak{n}_{v}^{\tau(\mathcal{S})+\left(m_{1}\left(m_{1}-1\right) / 2\right)\left(n_{1}-1\right)+\epsilon} \\
\operatorname{dim} \pi_{1, v}^{K_{1}(\mathfrak{n})_{v}} \prod_{i \geqslant 2}\left(\operatorname{dim} \pi_{i, v}^{K_{i}(\mathfrak{n})_{v}}\right)^{n_{i}}
\end{gathered}
$$

which gives (19).

5.3. $v$ split in $\boldsymbol{E} / \boldsymbol{F}, \boldsymbol{v} \in \boldsymbol{S}$. In this case, $G_{v}^{*} \simeq \operatorname{GL}\left(N, F_{v}\right) \simeq \operatorname{GL}\left(N, E_{w}\right)$ and $G_{v}$ is an inner form of $\operatorname{GL}\left(N, F_{v}\right)$. It is known (see [16, Theorem 1.6.4] for instance) that the packet $\Pi_{\psi_{v}}\left(G^{*}\right)$ contains exactly one element whereas $\Pi_{\psi_{v}}(G, \xi)$ has one or zero elements.

For $v \in S$, the constant $C_{v}$ can be arbitrary. This means that to prove (17), we need to know that the left hand side is bounded independently of $\psi_{v}$, and that if it is nonzero, then the right hand side is also nonzero. Both facts are provided by the following local lemma, where we consider $\psi_{v} \in \Psi\left(G_{v}^{*}\right)$ and bounded $\phi_{i, v} \in \Phi\left(\mathrm{GL}\left(m_{i}, F_{v}\right)\right)$ with $\psi_{v}=\bigoplus_{i=1}^{k} \phi_{i, v} \otimes v\left(n_{i}\right)$. The unique representations in $\Pi_{\psi_{v}}(G, \xi)$ and $\Pi_{\phi_{i, v}}\left(U\left(m_{i}\right)_{v}\right)=\Pi_{\phi_{i, v}}\left(\mathrm{GL}\left(m_{i}, F_{v}\right)\right)$ are denoted by $\pi_{v}$ and $\pi_{i, v}$, respectively.

Lemma 5.3. There is $C\left(K_{v}\right)>0$ such that $\operatorname{dim} \pi_{v}^{K_{v}} \leqslant C\left(K_{v}\right)$. For each $i$ there exists an open compact subgroup $K_{i, v} \subset U\left(m_{i}, F_{v}\right)$ depending only on $K_{v}$ such that the following is true for every $\psi_{v}$ and $\phi_{i, v}$ as above: if $\pi_{v}^{K_{v}} \neq 0$, then $\pi_{i, v}^{K_{i, v}} \neq 0$ for all $i$.

Proof. The first claim is Bernstein's uniform admissibility theorem [5]. (We need it just for unitary representations, but the proof there shows the theorem for irreducible admissible representations of general $p$-adic reductive groups.)

To prove the second claim, recall that $\psi_{v}$ gives rise to representations $\rho_{\psi_{v}}$ and $\pi_{\psi_{v}}$ of $G_{v}^{*} \simeq \mathrm{GL}\left(N, F_{v}\right)$ as in Section 5.1. So $\pi_{\psi_{v}}$ is an irreducible subquotient of $\rho_{\psi_{v}}$. 
The hypothesis $\pi_{v}^{K_{v}} \neq 0$ means that $1_{K_{v}}\left(\pi_{v}\right) \neq 0$. If we transfer $1_{K_{v}}$ to a function $1_{K_{v}}^{*}$ on $G_{v}^{*}$, we have the character identity $1_{K_{v}}^{*}\left(\pi_{\psi_{v}}\right)=e\left(G_{v}\right) a_{\psi_{v}} 1_{K_{v}}\left(\pi_{v}\right) \neq 0$ by [16, Theorem 1.6.4(1)] with certain signs $e\left(G_{v}\right), a_{\psi_{v}} \in\{ \pm 1\}$. If we let $K_{v}^{\prime} \subset$ $\mathrm{GL}\left(N, F_{v}\right)$ be an open compact subgroup such that $1_{K_{v}}^{*}$ is bi-invariant under $K_{v}^{\prime}$, this implies that $\pi_{\psi_{v}}^{K_{v}^{\prime}} \neq 0$ and thus $\rho_{\psi_{v}}^{K_{v}^{\prime}} \neq 0$. This gives $\pi_{i, v}^{K_{i, v}} \neq 0$ for suitable $K_{i, v} \subset \mathrm{GL}\left(m_{i}, F_{v}\right)$, which implies the claim. (To see this, one uses a description of invariant vectors in an induced representation under an open compact subgroup as in the first display of $[4, \mathrm{p} .26]$, noting that the double coset space $P \backslash G / K$ there is finite.)

5.4. $\boldsymbol{v}$ nonsplit in $\boldsymbol{E} / \boldsymbol{F}, \boldsymbol{v} \notin S$. In this case, for each $\psi_{v} \in \Psi\left(G_{v}^{*}\right)$ we have $\psi_{v}^{N}=\eta_{\chi_{\kappa}} \circ \psi_{v} \in \Psi(\tilde{G}(N))=\Psi\left(\operatorname{GL}\left(N, E_{w}\right)\right)$. This gives rise to representations $\pi_{\psi_{v}^{N}}$ and $\rho_{\psi_{v}^{N}}$ of $\operatorname{GL}\left(N, E_{w}\right)$ as in Section 5.1. Similarly $\phi_{i, v} \in \Phi\left(U\left(m_{i}\right)_{v}\right)$ gives a representation $\pi_{\phi_{i, v}^{m_{i}}}$ of $\operatorname{GL}\left(m_{i}, E_{w}\right)$ for the parameter $\eta_{\chi_{\kappa_{i}}} \circ \phi_{i, v}$. If $\psi_{v}$ and $\phi_{i, v}$ arise from global data as at the start of Section 5 then $\pi_{\phi_{i, v}^{m_{i}}}$ is nothing but $\mu_{i, w}$.

Inequality (17) in this case follows from the lemma below.

LEMMA 5.4. Consider $\psi_{v} \in \Psi\left(G_{v}^{*}\right)$ and $\phi_{i, v} \in \Phi\left(U\left(m_{i}\right)_{v}\right)$ as above such that $\psi_{v}^{N}=\bigoplus_{i \geqslant 1} \phi_{i, v}^{m_{i}} \otimes v\left(n_{i}\right)$. Then we have

$$
\sum_{\pi_{v} \in \Pi_{\psi_{v}}(G, \xi)} \operatorname{dim} \pi_{v}^{K_{v}} \leqslant 1
$$

If equality holds, then

$$
\sum_{\pi_{i, v} \in \Pi_{\phi_{i, v}}\left(U\left(m_{i}\right)\right)} \operatorname{dim} \pi_{i, v}^{K_{i, v}}=1
$$

for all $i$.

Proof. Suppose first that $s_{\psi_{v}} \in\{ \pm 1\}$. We have a hyperspecial subgroup $\widetilde{K}_{v}$ of $G(N)_{v} \simeq \mathrm{GL}\left(N, E_{w}\right)$. The twisted fundamental lemma implies that the functions $1_{K_{v}}$ and $1_{\widetilde{K}_{v} \rtimes \theta}$ are related by transfer.

Applying the character identity for $U(N)$ [25, Theorem 3.2.1(b)] with $s=1$ gives

$$
1_{K_{v}}^{U(N)}\left(\psi_{v}\right)=\sum_{\pi_{v} \in \Pi_{\psi_{v}}(G, \xi)} \operatorname{dim} \pi_{v}^{K_{v}},
$$

and combining this with the twisted character identity [25, Theorem 3.2.1(a)] and 
the twisted fundamental lemma gives

$$
\sum_{\pi_{v} \in \Pi_{\psi_{v}}(G, \xi)} \operatorname{dim} \pi_{v}^{K_{v}}=\operatorname{tr}\left(\widetilde{\pi}_{\psi_{v}^{N}}\left(1_{\widetilde{K}_{v} \rtimes \theta}\right)\right) .
$$

The twisted trace $\operatorname{tr}\left(\widetilde{\pi}_{\psi_{v}^{N}}\left(1_{\widetilde{K}_{v} \rtimes \theta}\right)\right)$ is equal to the trace of $\widetilde{\pi}_{\psi_{v}^{N}}(\theta)$ on $\pi_{\psi_{v}^{N}}^{\widetilde{K}_{v}}$, so we have

$$
\operatorname{tr}\left(\widetilde{\pi}_{\psi_{v}^{N}}\left(1_{\widetilde{K}_{v} \rtimes \theta}\right)\right) \leqslant \operatorname{dim} \pi_{\psi_{v}^{N}}^{\widetilde{K}_{v}}
$$

Since $\pi_{\psi_{v}^{N}}$ is a subquotient of $\rho_{\psi_{v}^{N}}$, we have

$$
\operatorname{dim} \pi_{\psi_{v}^{N}}^{\widetilde{K}_{v}} \leqslant \operatorname{dim} \rho_{\psi_{v}^{N}}^{\widetilde{K}_{v}} \leqslant 1
$$

which gives (20).

If equality holds, then $\psi_{v}^{N}$ is unramified. So all $\phi_{i, v}$ are unramified as well. Applying [25, Theorem 3.2.1(b)] to the parameter $\phi_{i, v}$ and the function $1_{K_{i, v}}$ for $U\left(m_{i}\right)$ gives

$$
\sum_{\pi_{i, v} \in \Pi_{\phi_{i, v}}\left(U\left(m_{i}\right)\right)} \operatorname{dim} \pi_{i, v}^{K_{i, v}}=1_{K_{i, v}}^{U\left(m_{i}\right)}\left(\phi_{i, v}\right) .
$$

If $\widetilde{\pi}_{\phi_{i, v}^{m_{i}}}$ is the canonical extension of $\pi_{\phi_{i, v}^{m_{i}}}$ to $\widetilde{G}\left(m_{i}\right)_{v}$ (via Whittaker normalization),

$$
\sum_{\pi_{i, v} \in \Pi_{\phi_{i, v}}\left(U\left(m_{i}\right)\right)} \operatorname{dim} \pi_{i, v}^{K_{i, v}}=\operatorname{tr}\left(\widetilde{\pi}_{\phi_{i, v}^{m_{i}}}\left(1_{\widetilde{K}_{i, v} \rtimes \theta}\right)\right) .
$$

$\operatorname{tr}\left(\widetilde{\pi}_{\phi_{i, v}^{m_{i}}}\left(1_{\widetilde{K}_{i, v} \rtimes \theta}\right)\right)$ is the trace of $\theta$ on the one-dimensional space $\pi_{\phi_{i, v}^{m_{i}}}$, so we have $\operatorname{tr}\left(\widetilde{\pi}_{\phi_{i, v}^{m_{i}}}\left(1_{\widetilde{K}_{i, v} \rtimes \theta}\right)\right)= \pm 1$, and (21) follows from positivity.

Now suppose that $s_{\psi_{v}} \notin\{ \pm 1\}$, and let $\left(G^{\mathfrak{e}}, s^{\mathfrak{e}}, \eta^{\mathfrak{e}}\right)$ be the elliptic endoscopic triple for $G$ with $s^{\mathfrak{e}}=s_{\psi_{v}}$. We have $G^{\mathfrak{e}}=U(a) \times U(b)$ for some $a, b>0$. There is an Arthur parameter $\psi^{\mathfrak{e}}$ for $G^{\mathfrak{e}}$ such that $\eta^{\mathfrak{e}} \circ \psi^{\mathfrak{e}}=\psi$, which we may factorize as $\psi^{\mathfrak{e}}=\psi_{1} \times \psi_{2}$. We let $K_{v}^{\mathfrak{e}} \subset G^{\mathfrak{e}}\left(F_{v}\right)$ be a hyperspecial subgroup, and let $1_{K_{v}}^{\mathfrak{e}}$ be the characteristic function of $K_{v}^{\mathfrak{e}}$. The Fundamental Lemma implies that $1_{K_{v}} \in \mathcal{H}\left(G_{v}\right)$ and $1_{K_{v}}^{\mathfrak{e}} \in \mathcal{H}\left(G_{v}^{\mathfrak{e}}\right)$ have $\Delta[\mathfrak{e}, \xi, z]$-matching orbital integrals. Applying [25, Theorem 3.2.1(b)] with $s=s_{\psi_{v}}$ gives

$$
\sum_{\pi_{v} \in \Pi_{\psi_{v}}(G, \xi)} 1_{K_{v}}\left(\pi_{v}\right)=1_{K_{v}}^{\mathfrak{e}}\left(\psi_{v}^{\mathfrak{e}}\right)=1_{K_{1, v}}\left(\psi_{1, v}\right) 1_{K_{2, v}}\left(\psi_{2, v}\right) .
$$

The result now follows by applying the result in the case $s_{\psi_{v}} \in\{ \pm 1\}$ to the groups $U(a)$ and $U(b)$. 
5.5. $\boldsymbol{v}$ nonsplit in $\boldsymbol{E} / \boldsymbol{F}, \boldsymbol{v} \in \boldsymbol{S}$. Here we prove a result analogous to Lemma 5.3.

LEMMA 5.5. There exist open compact subgroups $K_{i, v} \subset U\left(m_{i}\right)_{v}$ depending only on $K_{v}$ such that the following holds: given $\psi_{v} \in \Psi\left(G_{v}\right)$ and $\phi_{i, v} \in \Phi\left(U\left(m_{i}\right)_{v}\right)$ such that $\psi_{v}^{N}=\bigoplus_{i=1}^{k} \phi_{i, v}^{N} \otimes v\left(n_{i}\right)$ (thus $\phi_{i, v}$ are bounded), if

$$
\sum_{\pi_{v} \in \Pi_{\psi_{v}}(G, \xi)} \operatorname{dim} \pi_{v}^{K_{v}} \neq 0 \text { then } \sum_{\pi_{i, v} \in \Pi_{\phi_{i, v}}\left(U\left(m_{i}\right)\right)} \operatorname{dim} \pi_{i, v}^{K_{i, v}} \neq 0 .
$$

Moreover there is a constant $C\left(K_{v}\right)>0$ which is independent of $\psi_{v}$ such that

$$
\sum_{\pi_{v} \in \Pi_{\psi_{v}}(G, \xi)} \operatorname{dim} \pi_{v}^{K_{v}} \leqslant C\left(K_{v}\right)
$$

Proof. We begin with the first claim. Suppose $s_{\psi_{v}} \in\{ \pm 1\}$. Let $1_{K_{v}}^{*}$ be the transfer of $1_{K_{v}}$ to $G_{v}^{*}$. The character identity of [16, Theorem 1.6.1(4)] gives

$$
0 \neq e\left(G_{v}\right) \sum_{\pi_{v} \in \Pi_{\psi_{v}}(G, \xi)} \operatorname{dim} \pi_{v}^{K_{v}}=1_{K_{v}}^{*}\left(\psi_{v}\right)
$$

where $e\left(G_{v}\right) \in\{ \pm 1\}$; note that the coefficients $\langle\pi, 1\rangle$ appearing in the cited theorem are all 1 (where we take $s^{\mathfrak{e}}=s_{\psi_{v}}$ ). Using the surjectivity result of Mok [25, Proposition 3.1.1(b)], there is a function $\widetilde{1}_{K_{v}}$ on $\widetilde{G}(N)_{v}$ whose twisted transfer to $G_{v}^{*}$ is $1_{K_{v}}^{*}$, and so we have $1_{K_{v}}^{*}\left(\psi_{v}\right)=\operatorname{tr}\left(\widetilde{\pi}_{\psi_{v}}\left(\widetilde{1}_{K_{v}}\right)\right)$. Let $\widetilde{K}_{v} \subset G(N)_{v}$ be a compact open subgroup such that $\widetilde{1}_{K_{v}}$ is bi-invariant under $\widetilde{K}_{v}$. It follows that we must have $\pi_{\psi_{v}}^{\widetilde{K}_{v}} \neq 0$, and hence there are compact open $\widetilde{K}_{i, v} \subset G\left(m_{i}\right)_{v}$ depending only on $K_{v}$ such that $\pi_{\phi_{i, v}}^{\widetilde{K}_{i, v}} \neq 0$. The result now follows from Lemma 5.6.

Now suppose that $s_{\psi_{v}} \notin\{ \pm 1\}$, and let $\left(G^{\mathfrak{e}}, s^{\mathfrak{e}}, \eta^{\mathfrak{e}}\right)$ be the elliptic endoscopic triple for $G$ with $s^{\mathfrak{e}}=s_{\psi_{v}}$ and so $G^{\mathfrak{e}}=U(a) \times U(b)$ for some $a, b>0$. There is an Arthur parameter $\psi^{\mathfrak{e}}$ for $G^{\mathfrak{e}}$ such that $\eta^{\mathfrak{e}} \circ \psi^{\mathfrak{e}}=\psi$, which we may factorize as $\psi^{\mathfrak{e}}=\psi_{1} \times \psi_{2}$. Let $1_{K_{v}}^{\mathfrak{e}}$ be the function obtained by transferring $1_{K_{v}}$ to $G_{v}^{\mathfrak{e}}$. Applying the trace identity

$$
e\left(G_{v}\right) \sum_{\pi_{v} \in \Pi_{\psi_{v}}(G, \xi)} \operatorname{dim} \pi_{v}^{K_{v}}=1_{K_{v}}^{\mathfrak{e}}\left(\psi_{v}^{\mathfrak{e}}\right)
$$

gives $1_{K_{v}}^{\mathfrak{e}}\left(\psi_{v}^{\mathfrak{e}}\right) \neq 0$. Because $1_{K_{v}}^{\mathfrak{e}}\left(\psi_{v}^{\mathfrak{e}}\right)$ is equal to a sum of traces there is a compact open $K_{1, v} \times K_{2, v} \subset G_{v}^{\mathfrak{e}}$ such that

$$
1_{K_{1, v} \times K_{2, v}}\left(\psi_{v}^{\mathfrak{e}}\right)=1_{K_{1, v}}\left(\psi_{1, v}\right) 1_{K_{2, v}}\left(\psi_{2, v}\right) \neq 0
$$


and the result now follows from the case $s_{\psi_{v}} \in\{ \pm 1\}$ for the groups $U(a)$ and $U(b)$.

We now prove the second claim. Suppose $s_{\psi_{v}} \in\{ \pm 1\}$. We again use the identity

$$
e\left(G_{v}\right) \sum_{\pi_{v} \in \Pi_{\psi_{v}}(G, \xi)} \operatorname{dim} \pi_{v}^{K_{v}}=\operatorname{tr}\left(\tilde{\pi}_{\psi_{v}}\left(\tilde{1}_{K_{v}}\right)\right),
$$

and let $\widetilde{K}_{v} \subset G(N)_{v}$ be a compact open subgroup such that $\tilde{1}_{K_{v}}$ is bi-invariant under $\widetilde{K}_{v}$. The trace $\operatorname{tr}\left(\widetilde{\pi}_{\psi_{v}}\left(\widetilde{1}_{K_{v}}\right)\right)$ is equal to the trace of $\widetilde{\pi}_{\psi_{v}}\left(\widetilde{1}_{K_{v}}\right)$ on the space $\pi_{\psi_{v}}^{\widetilde{K}_{v}}$, and the operator norm of $\widetilde{\pi}_{\psi_{v}}\left(\tilde{1}_{K_{v}}\right)$ is at most $\left\|\tilde{1}_{K_{v}}\right\|_{1}=C\left(K_{v}\right)$. We therefore have $\left|\operatorname{tr}\left(\widetilde{\pi}_{\psi_{v}}\left(\tilde{1}_{K_{v}}\right)\right)\right| \leqslant C\left(K_{v}\right) \operatorname{dim} \pi_{\psi_{v}}^{\widetilde{K}_{v}}$, and the result follows as in Lemma 5.3. If $s_{\psi_{v}} \notin\{ \pm 1\}$, we reduce to the case of $U(a) \times U(b)$ as before.

Recall that $\eta_{\chi_{\kappa_{i}}} \circ \phi_{i, v} \in \Phi\left(G\left(m_{i}\right)_{v}\right)$ corresponds to $\mu_{i, w}$ via local Langlands under the isomorphism $G\left(m_{i}\right)_{v} \simeq \operatorname{GL}\left(m_{i}, E_{w}\right)$, where $w$ is the unique place of $E$ above $v$.

LEMMA 5.6. If $\widetilde{K}_{i, w} \subset \mathrm{GL}\left(m_{i}, E_{w}\right)$ is a compact open subgroup, then there is a compact open subgroup $K_{i, v} \subset U\left(m_{i}\right)_{v}$ with the following property: For any bounded parameter $\phi_{i, v} \in \Phi\left(U\left(m_{i}\right)_{v}\right)$ and the representation $\mu_{i, w}$ of $\operatorname{GL}\left(m_{i}, E_{w}\right)$ corresponding as above, if $\mu_{i, w}^{\widetilde{K}_{i, w}} \neq 0$ then

$$
\sum_{\pi_{i, v} \in \Pi_{\phi_{i, v}}\left(U\left(m_{i}\right)\right)} \operatorname{dim} \pi_{i, v}^{K_{i, v}} \neq 0 .
$$

Proof. The only nontrivial part of the lemma is the assertion that $K_{i, v}$ may be chosen independently of $\mu_{i, w}$. To this end, we will show that $\phi_{i, v}\left(\right.$ or $\left.\mu_{i, w}\right)$ varies over a compact domain and that $K_{i, v}$ as in the lemma can be chosen in open neighborhoods. Then the proof will be complete by taking intersection of the finitely many $K_{i, v}$ for a finite open covering.

By a theorem of Jacquet, our assumption that $\mu_{i, w}$ was tempered implies that $\mu_{i, w}$ belongs to a family of full induced representations from some

$$
\mu_{1}^{\prime}|\cdot|^{i s_{1}} \otimes \cdots \otimes \mu_{k}^{\prime}|\cdot|^{i s_{k}}
$$

with $\mu_{j}^{\prime}$ square integrable and $s_{j} \in \mathbb{R} /\left(2 \pi / \log q_{w}\right) \mathbb{Z}$. Our assumption that $\mu_{i, w}$ had bounded depth implies that the set of tuples $\mu_{1}^{\prime}, \ldots, \mu_{k}^{\prime}$ we must consider is finite, and so we only need to consider one. We then need to show that the set of $s_{j}$ such that $\mu_{i, w}$ is conjugate self-dual (that is $\mu_{i, w} \simeq \mu_{i, w} \circ \theta$ ) is compact. Because $\mu_{j}^{\prime}|\cdot|^{i s_{j}} \circ \theta=\left(\mu_{j}^{\prime} \circ \theta\right)|\cdot|^{-i s_{j}}$, this condition is equivalent to saying that the multisets $\left\{\mu_{j}^{\prime}|\cdot|^{i s_{j}}\right\}$ and $\left\{\left(\mu_{j}^{\prime} \circ \theta\right)|\cdot|^{-i s_{j}}\right\}$ are equivalent. This in turn is equivalent to the 
existence of a permutation $\sigma \in S_{k}$ such that $\left.\mu_{j}^{\prime}|\cdot|{ }^{i s_{j}} \simeq\left(\mu_{\sigma(j)}^{\prime} \circ \theta\right)|\cdot|\right|^{-i s_{\sigma(j)}}$ for all $j$. For each $\sigma$ the set of $s_{j}$ satisfying this is closed, and hence the set of $s_{j}$ such that $\mu_{i, w}$ is conjugate self-dual is closed and compact.

For fixed $s_{1}, \ldots, s_{k}$, there is some $K_{i, v}$ such that $1_{K_{i, v}}\left(\phi_{i, v}\right) \neq 0$, where $1_{K_{i, v}}\left(\phi_{i, v}\right)$ is equal to the left hand side of equation (22) by definition. Also, if we transfer $1_{K_{i, v}}$ to $\widetilde{1}_{K_{i, v}}$ on $\widetilde{G}\left(m_{i}\right)$ using the surjectivity theorem of Mok [25, Proposition 3.1.1(b)] then the character identity tells us that $1_{K_{i, v}}\left(\phi_{i, v}\right)=\operatorname{tr}\left(\widetilde{\pi}_{\phi_{i, v}}^{m_{i}}\left(\widetilde{1}_{K_{i, v}}\right)\right.$ ) (where the twisted trace is Whittaker normalized). The point is that $\operatorname{tr}\left(\widetilde{\pi}_{\phi_{i, v}}^{m_{i}}\left(\widetilde{1}_{K_{i, v}}\right)\right)$ varies continuously in the $s_{j}$ (see [27]) so we still have $1_{K_{i, v}}\left(\phi_{i, v}\right) \neq 0$ around an open neighborhood of $s_{1}, \ldots, s_{k}$ (where $\phi_{i, v}$ varies as $s_{1}, \ldots, s_{k}$ vary). The result now follows by compactness.

\section{Archimedean control on parameters}

In this section, we prove some useful conditions on the parameters $\psi$ that contribute to the cohomology of $X(\mathfrak{n})$.

Given $\phi_{\infty}=\otimes_{v \mid \infty} \phi_{v} \in \Phi\left(U(n)_{\infty}\right)$ for $n \geqslant 1$, note that the restriction of $\phi_{v}$ to $W_{\mathbb{C}}=\mathbb{C}^{\times}$(for a fixed isomorphism $\bar{F}_{v} \simeq \mathbb{C}$ ), viewed as an $n$-dimensional representation via $\widehat{U(n)}=\mathrm{GL}(n, \mathbb{C})$, is a direct sum of $n$ characters $z \mapsto z^{a_{i, v}} \bar{z}^{b_{i, v}}$ with $a_{i, v}, b_{i, v} \in \mathbb{C}$ and $a_{i, v}-b_{i, v} \in \mathbb{Z}$ for $i=1, \ldots, n$. We say that $\phi_{\infty}$ is $\mathrm{C}$ algebraic if $n$ is odd and all $a_{i, v} \in \mathbb{Z}$ or if $n$ is even and all $a_{i, v} \in \frac{1}{2}+\mathbb{Z}$. We say $\phi_{v}$ is regular if $a_{i, v}$ are distinct. If $\pi_{\infty}$ is a member of the $L$-packet for $\phi_{\infty}$ then $\pi_{\infty}$ is said to be regular or C-algebraic if $\phi_{\infty}$ is. (This is Clozel's definition and coincides with the general definition [8, Definition 2.3.3] for general reductive groups.)

Let $\left.\mathcal{S}=\left(n_{1}, m_{1}\right), \ldots, \underset{\sim}{\sim} \tilde{n}_{k}, m_{k}\right)$ be a shape as in Section 5. If $\psi \in \Psi_{2}\left(G^{*}, \eta_{\chi_{k}}\right)_{\mathcal{S}}$ then $\psi$ gives rise to $\mu_{i} \in \widetilde{\Phi}_{\text {sim }}\left(m_{i}\right)$ and $\phi_{i} \in \Phi_{\text {sim }}\left(U\left(m_{i}\right), \eta_{\chi_{\kappa_{i}}}\right)$ as before.

LEMMA 6.1. Let $\psi \in \Psi_{2}\left(G^{*}, \eta_{\chi_{\kappa}}\right)_{\mathcal{S}}$. If there is $\pi_{\infty} \in \Pi_{\psi_{\infty}}(G, \xi)$ with $h^{d}\left(\mathfrak{g}, K_{\infty} ; \pi_{\infty}\right) \neq 0$ then $\phi_{i, \infty}$ or $\phi_{i, \infty} \otimes \chi_{-, \infty}$ is C-algebraic. Moreover every $\phi_{i, v}$ is bounded at every place $v$ (equivalently $\mu_{i, v}$ is tempered at every place $v$ ).

Proof. Since $\pi_{\infty}$ contributes to cohomology, its infinitesimal character is equal to that of the trivial representation. In particular it is regular $\mathrm{C}$-algebraic, cf. [8, Lemmas 7.2.2, 7.2.3]. Hence $\phi_{\psi_{\infty}}$ is regular C-algebraic. (Here we use the simple recipe to determine the infinitesimal character of $\pi_{\infty}$ from $\phi_{\psi_{\infty}}$ by differentiation, as described in [22, Section 2.1].) For each infinite place $v$, the representation $\left.\phi_{i, v}\right|_{W_{\mathbb{C}}}$ is the direct sum of $m_{i}$ characters, say $\eta_{i, 1}, \ldots, \eta_{i, m_{i}}$. Then $\bigoplus_{j=1}^{m_{i}} \bigoplus_{l=0}^{n_{i}-1} \eta_{i, j}|\cdot|^{\left(n_{i}-1\right) / 2-l}$ appears as a subrepresentation of $\phi_{\psi_{\infty}}$. As the latter is regular C-algebraic, we see that for each $v\left|\infty, \phi_{i, v}\right|_{W_{\mathbb{C}}}$ is regular and that either 
$\left.\phi_{i, v}\right|_{W_{\mathbb{C}}}$ or $\left.\phi_{i, v}\right|_{W_{\mathbb{C}}} \otimes|\cdot|^{1 / 2}$ is C-algebraic, depending on the parity of $N-n_{i}$. It follows that $\left.\phi_{i, \infty}\right|_{W_{\mathbb{C}}}$ or $\left.\phi_{i, \infty}\right|_{W_{\mathbb{C}}} \otimes|\cdot|{ }^{1 / 2}$ is regular C-algebraic. By the definition of $\chi_{-}$in Section 2, $\left.\phi_{i, \infty}\right|_{W_{\mathbb{C}}} \otimes|\cdot|^{1 / 2}$ is C-algebraic if and only if $\left.\phi_{i, \infty}\right|_{W_{\mathbb{C}}} \otimes \chi_{-, \infty}$ is.

The key point is that $\mu_{i}$ or $\mu_{i} \otimes \chi_{-}$is an automorphic representation with regular $\mathrm{C}$-algebraic component at $\infty$ (recalling that $\mu_{i, \infty}$ lies in the packet for $\left.\phi_{i, \infty}\right)$. Both $\mu_{i}$ and $\mu_{i} \otimes \chi_{-}$are cuspidal and conjugate self-dual, so either $\mu_{i}$ or $\mu_{i} \otimes \chi_{-}$(whichever is C-algebraic at $\infty$ ) is essentially tempered at all finite places by [9, Theorem 1.2] (the cohomological condition in [9] follows from regular C-algebraicity, cf. [11, Lemme 3.14]) and at all infinite places by [11, Lemme 4.9]. In either case, twisting by $\chi_{-}$if necessary, we deduce that $\mu_{i}$ is essentially tempered everywhere. Since the central character of $\mu_{i}$ is unitary, we see that $\mu_{i}$ is tempered everywhere. By the local Langlands correspondence [25, Theorem 2.5.1(b)], this is equivalent to $\phi_{i, v}$ being bounded at every $v$.

LEMMA 6.2. For each $i$, there is a finite set of parameters $\mathcal{P}_{i, \infty} \subset \Phi\left(U\left(m_{i}\right)_{\infty}\right)$ with the following property: If $\psi \in \Psi_{2}\left(G^{*}, \eta_{\chi_{\kappa}}\right)_{\mathcal{S}}$, and there exists $\pi_{\infty} \in \Pi_{\psi_{\infty}}$ $(G, \xi)$ with $h^{d}\left(\mathfrak{g}, K_{\infty} ; \pi_{\infty}\right) \neq 0$, then $\phi_{i, \infty} \in \mathcal{P}_{i, \infty}$.

Proof. The infinitesimal character of $\pi_{\infty}$ is determined by the condition that $h^{d}(\mathfrak{g}$, $\left.K_{\infty} ; \pi_{\infty}\right) \neq 0$ (to be the half sum of all positive roots of $G$ ), thus there are finitely many such $\pi_{\infty}$. So they are contained in finitely many Arthur packets, whose parameters form a finite subset $\mathcal{P} \subset \Psi\left(U(N)_{\infty}\right)$. If $\psi$ gives rise to $\phi_{i}$ then $\eta_{\chi_{\kappa_{i}}} \circ$ $\left(\phi_{i, v} \otimes v\left(n_{i}\right)\right)$ should appear as a factor of $\eta_{\chi_{\kappa}} \circ \psi_{v}$ for every infinite place $v$. Since we have the constraint $\otimes_{v \mid \infty} \psi_{v} \in \mathcal{P}$, it is clear that there are finitely many possibilities for $\phi_{i, \infty}$.

\section{Summing over parameters}

In this section we continue the proof of Theorem 1.2 from the end of Section 4 and finish the proof. In the preliminary bound (13), we will fix a shape $\mathcal{S}$ and bound the contribution to $h_{(2)}^{d}(X(\mathfrak{n}))$ from parameters in $\Psi_{2}\left(G^{*}, \eta_{\chi_{\kappa}}\right)_{\mathcal{S}}$, which we denote by $h_{(2)}^{d}(X(\mathfrak{n}))_{\mathcal{S}}$. Clearly it suffices to establish a bound for $h_{(2)}^{d}(X(\mathfrak{n}))_{\mathcal{S}}$ as in Theorem 1.2.

Suppose $\psi \in \Psi_{2}\left(G^{*}, \eta_{\chi_{\kappa}}\right)_{\mathcal{S}}$ has the property that there is $\pi \in \Pi_{\psi}(G, \xi)$ with $h^{d}\left(\mathfrak{g}, K_{\infty} ; \pi_{\infty}\right) \neq 0$. Proposition 3.1 implies that $\pi_{v_{0}}$ must be a Langlands quotient of a standard representation with an exponent of the form $(z / \bar{z})^{p / 2}(z \bar{z})^{(a-1) / 2}$ for some $a \geqslant N-d$. Proposition 13.2 of [3] implies that there is $i$ such that $n_{i} \geqslant N-d$, and we assume that this is $n_{1}$. Note that [3, Proposition 13.2] implicitly assumes 
that the other archimedean components of $\pi$ have regular infinitesimal character, but this is satisfied in our case.

Apply Lemma 6.2 to obtain finite sets $\mathcal{P}_{i, \infty} \subset \Phi\left(U\left(m_{i}\right)_{\infty}\right)$ for all $i$ such that if $\psi \in \Psi_{2}\left(G^{*}, \eta_{\chi_{\kappa}}\right)_{\mathcal{S}}$, and there is $\pi \in \Pi_{\psi}(G, \xi)$ with $h^{d}\left(\mathfrak{g}, K_{\infty} ; \pi_{\infty}\right) \neq 0$, then $\phi_{i, \infty} \in \mathcal{P}_{i, \infty}$. Let $\Psi_{\text {rel }}$ be the set of $A$-parameters $\psi \in \Psi_{2}\left(G^{*}, \eta_{\chi_{k}}\right)_{\mathcal{S}}$ with $\phi_{i}$ bounded everywhere and $\phi_{i, \infty} \in \mathcal{P}_{i, \infty}$ for all $i$. By Lemmas 6.1 and 6.2 we have

$$
h_{(2)}^{d}(X(\mathfrak{n}))_{\mathcal{S}} \leqslant \sum_{\psi \in \Psi_{\mathrm{rel}}} \sum_{\pi \in \Pi_{\psi}(G, \xi)} h^{d}\left(\mathfrak{g}, K_{\infty} ; \pi_{\infty}\right) \operatorname{dim} \pi_{f}^{K(\mathfrak{n})},
$$

and because $h^{d}\left(\mathfrak{g}, K_{\infty} ; \pi_{\infty}\right)$ is bounded we may simplify this to

$$
h_{(2)}^{d}(X(\mathfrak{n}))_{\mathcal{S}} \ll \sum_{\psi \in \Psi_{\mathrm{rel}}} \sum_{\pi \in \Pi_{\psi}(G, \xi)} \operatorname{dim} \pi_{f}^{K(\mathfrak{n})} .
$$

Because $\phi_{i}$ is bounded everywhere for every $i$, we may apply Proposition 5.1 to obtain

$$
h_{(2)}^{d}(X(\mathfrak{n}))_{\mathcal{S}} \ll \prod_{v \mid \mathfrak{n}}\left(1+1 / q_{v}\right)^{\sigma(\mathcal{S})} N \mathfrak{n}^{\tau(\mathcal{S})} \sum_{\psi \in \Psi_{\text {rel }}} \prod_{i \geqslant 1}\left(\sum_{\pi_{i} \in \Pi_{\phi_{i}}\left(U\left(m_{i}\right)\right)} \operatorname{dim} \pi_{i, f}^{K_{i}(\mathfrak{n})}\right)^{n_{i}} .
$$

Let $\Phi_{\text {sim }}^{\text {bdd }}\left(U\left(m_{i}\right), \eta_{\chi_{\kappa_{i}}}\right)$ denote the set of simple parameters that are bounded everywhere. Taking a sum over $\psi \in \Psi_{\text {rel }}$ corresponds to taking a sum over the possibilities for $\phi_{i} \in \Phi_{\text {sim }}^{\text {bdd }}\left(U\left(m_{i}\right), \eta_{\chi_{\kappa_{i}}}\right)$ with $\phi_{i, \infty} \in \mathcal{P}_{i, \infty}$. We may therefore factorize the sum over $\psi$ to ones over $\phi_{i}$, which gives

$$
\begin{aligned}
& h_{(2)}^{d}(X(\mathfrak{n}))_{\mathcal{S}} \ll \prod_{v \mid \mathfrak{n}}\left(1+1 / q_{v}\right)^{\sigma(\mathcal{S})} N \mathfrak{n}^{\tau(\mathcal{S})}
\end{aligned}
$$

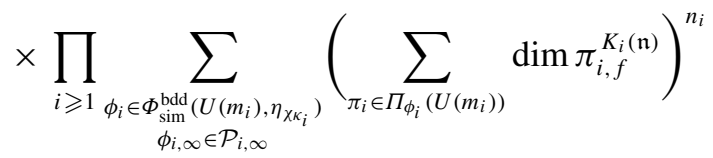

$$
\begin{aligned}
& \leqslant \prod_{v \mid \mathfrak{n}}\left(1+1 / q_{v}\right)^{\sigma(\mathcal{S})} N \mathfrak{n}^{\tau(\mathcal{S})} \\
& \times \prod_{i \geqslant 1}\left(\sum_{\substack{\phi_{i} \in \Phi_{\text {sim }}^{\text {bdd }} \\
\phi_{i, \infty} \in \mathcal{P}_{i, \infty}}} \sum_{\substack{\left.\left.m_{i}\right), \eta_{\chi \kappa_{i}}\right) \\
\pi_{i} \in \Pi_{\phi_{i}}\left(U\left(m_{i}\right)\right)}} \operatorname{dim} \pi_{i, f}^{K_{i}(\mathfrak{n})}\right)^{n_{i}} .
\end{aligned}
$$

We may bound the sums using the global limit multiplicity formula of Savin [29]. Indeed, because $\phi_{i}$ is a simple generic parameter, the packet $\Pi_{\phi_{i}}\left(U\left(m_{i}\right)\right)$ is stable, so that every representation $\pi_{i} \in \Pi_{\phi_{i}}\left(U\left(m_{i}\right)\right)$ occurs in the discrete 
spectrum of $U\left(m_{i}\right)$ with multiplicity one. In fact, $\pi_{i}$ must actually lie in the cuspidal spectrum by [32, Theorem 4.3], because $\pi_{i, \infty}$ is tempered. Because the archimedean components of $\phi_{i}$ are restricted to finite sets, there is a finite set $\Pi_{i, \infty}$ of representations of $U\left(m_{i}\right)_{\infty}$ such that if $\pi_{i} \in \Pi_{\phi_{i}}\left(U\left(m_{i}\right)\right)$ then $\pi_{i, \infty} \in \Pi_{i, \infty}$. If $m_{\text {cusp }}\left(\pi_{\infty}, Y_{i}(\mathfrak{n})\right)$ denotes the multiplicity with which an irreducible representation $\pi_{\infty}$ of $U\left(m_{i}\right)_{\infty}$ occurs in the $L^{2}$-space of cusp forms $L_{\text {cusp }}^{2}\left(Y_{i}(\mathfrak{n})\right)$, where $Y_{i}(\mathfrak{n})=$ $U\left(m_{i}, F\right) \backslash U\left(m_{i}, \mathbb{A}\right) / K_{i}(\mathfrak{n})$, we have

$$
\begin{aligned}
\sum_{\substack{\phi_{i} \in \Phi_{\text {sim }}^{\text {bdd }}\left(U\left(m_{i}\right), \eta_{\chi_{K_{i}}}\right) \\
\phi_{i, \infty} \in \mathcal{P}_{i, \infty} \in \Pi_{\phi_{i}}\left(U\left(m_{i}\right)\right)}} \operatorname{dim} \pi_{i, f}^{K_{i}(\mathfrak{n})} & \leqslant \sum_{\substack{\pi_{i} \subset L_{\text {cusp }}^{2}\left(\left[U\left(m_{i}\right)\right]\right) \\
\pi_{i, \infty} \in \Pi_{i, \infty}}} \operatorname{dim} \pi_{i, f}^{K_{i}(\mathfrak{n})} \\
& \left.=\sum_{\substack{\pi_{\infty} \in \Pi_{i, \infty} \\
m_{\text {cusp }}}} \sum_{\infty}, Y_{i}(\mathfrak{n})\right) .
\end{aligned}
$$

For each $\pi_{\infty}$, Savin [29] gives

$$
m_{\text {cusp }}\left(\pi_{\infty}, Y_{i}(\mathfrak{n})\right) \ll\left[K_{i}: K_{i}(\mathfrak{n})\right] \ll \prod_{v \mid \mathfrak{n}}\left(1-1 / q_{v}\right) N \mathfrak{n}^{m_{i}^{2}},
$$

and combining this with (24) gives

$$
h_{(2)}^{d}(X(\mathfrak{n}))_{\mathcal{S}} \ll \prod_{v \mid \mathfrak{n}}\left(1-1 / q_{v}\right) N \mathfrak{n}^{\tau^{\prime}(\mathcal{S})},
$$

where $\tau^{\prime}(\mathcal{S})=\tau(\mathcal{S})+\sum_{i \geqslant 1} n_{i} m_{i}^{2}$. If $1 \leqslant m_{1} \leqslant 3$ then applying Proposition 5.1 and working as above gives

$$
h_{(2)}^{d}(X(\mathfrak{n}))_{\mathcal{S}} \ll \prod_{v \mid \mathfrak{n}}\left(1+1 / q_{v}\right)^{\sigma_{l}^{\prime}(\mathcal{S})} N \mathfrak{n}^{\tau_{l}^{\prime}(\mathcal{S})},
$$

where $l=m_{1}, \tau_{l}^{\prime}(\mathcal{S})=\tau_{l}(\mathcal{S})+m_{1}^{2}+\sum_{i \geqslant 2} n_{i} m_{i}^{2}$, and $\sigma_{l}^{\prime}(\mathcal{S})=\sigma_{l}(\mathcal{S})-1-\sum_{i \geqslant 2} n_{i}$.

The bounds for the functions $\tau^{\prime}$ and $\tau_{j}^{\prime}$ given by Lemma 7.1 then imply that

$$
h_{(2)}^{d}(X(\mathfrak{n}))_{\mathcal{S}} \ll_{\epsilon} N \mathfrak{n}^{N d+\epsilon},
$$

unless we are in one of the two cases listed there. In the exceptional case (ii) we have $h_{(2)}^{d}(X(\mathfrak{n}))_{\mathcal{S}} \ll N \mathfrak{n}^{N d+1+\epsilon}$, and in case (i), which should give the general main term, we have

$$
h_{(2)}^{d}(X(\mathfrak{n}))_{\mathcal{S}} \ll \prod_{v \mid \mathfrak{n}}\left(1-1 / q_{v}\right) N \mathfrak{n}^{N d+1} .
$$

This completes the proof of Theorem 1.2.

It remains to prove the lemma used in the above proof. 
LEMMA 7.1. If $m_{1} \geqslant 4$, we have $\tau^{\prime}(\mathcal{S}) \leqslant N d$. If $m_{1}=l, l=1,2,3$, we have $\tau_{l}^{\prime}(\mathcal{S}) \leqslant N d+\epsilon$, except in the following cases.

(i) $\mathcal{S}=(N-d, 1),(1, d)$, in which case $\tau_{1}^{\prime}(\mathcal{S})=N d+1$.

(ii) $\mathcal{S}=(2,2)$ and $d=2$, in which case $\tau_{2}^{\prime}(\mathcal{S})=N d+1+\epsilon=9+\epsilon$.

Proof. We begin with the case $m_{1} \geqslant 4$. The inequality $d \geqslant N-n_{1}$ implies that it suffices to prove $\tau^{\prime}(\mathcal{S}) \leqslant N\left(N-n_{1}\right)$. Substituting the definition of $\tau^{\prime}$ and simplifying, we must show that

$$
\left(\begin{array}{l}
N \\
2
\end{array}\right)+\sum_{i \geqslant 1} n_{i}\left(\begin{array}{c}
m_{i}+1 \\
2
\end{array}\right) \leqslant N\left(N-n_{1}\right) .
$$

We next eliminate the variables other than $N, m_{1}$ and $n_{1}$. The identity $\left(\begin{array}{c}n+1 \\ 2\end{array}\right)=$ $1+\cdots+n$ implies that if $A=\sum a_{i}$, then $\left(\begin{array}{c}A+1 \\ 2\end{array}\right) \geqslant \sum\left(\begin{array}{c}a_{i}+1 \\ 2\end{array}\right)$, and applying this to the $m_{i}$ with multiplicity $n_{i}$ for $i \geqslant 2$ gives

$$
\sum_{i \geqslant 2} n_{i}\left(\begin{array}{c}
m_{i}+1 \\
2
\end{array}\right) \leqslant\left(\begin{array}{c}
N-n_{1} m_{1}+1 \\
2
\end{array}\right) .
$$

Note that equality occurs above if and only if $\sum_{i \geqslant 2} n_{i}$ is either 0 or 1 . After applying this in (25), we are reduced to showing that

$$
\left(\begin{array}{l}
N \\
2
\end{array}\right)+n_{1}\left(\begin{array}{c}
m_{1}+1 \\
2
\end{array}\right)+\left(\begin{array}{c}
N-n_{1} m_{1}+1 \\
2
\end{array}\right) \leqslant N\left(N-n_{1}\right) .
$$

Simplifying gives

$$
\begin{gathered}
N(N-1)+n_{1}\left(m_{1}+1\right) m_{1}+\left(N-n_{1} m_{1}+1\right)\left(N-n_{1} m_{1}\right) \leqslant 2 N\left(N-n_{1}\right) \\
-2 m_{1} n_{1} N+2 N n_{1}+m_{1}^{2} n_{1}^{2} \leqslant-m_{1}^{2} n_{1} \\
0 \leqslant 2 m_{1} N-2 N-m_{1}^{2} n_{1}-m_{1}^{2} .
\end{gathered}
$$

As $N \geqslant m_{1} n_{1}$, we have $m_{1} N \geqslant m_{1}^{2} n_{1}$ so that

$$
2 m_{1} N-2 N-m_{1}^{2} n_{1}-m_{1}^{2} \geqslant\left(m_{1}-2\right) N-m_{1}^{2} .
$$

Because $n_{1} \geqslant 2$ we have $N \geqslant n_{1} m_{1} \geqslant 2 m_{1}$, so that

$$
\left(m_{1}-2\right) N-m_{1}^{2} \geqslant m_{1}^{2}-4 m_{1} \geqslant 0,
$$

where we have used $m_{1} \geqslant 4$ at the last step. 
In the case $m_{1}=1$, we have

$$
\tau_{1}^{\prime}(\mathcal{S})=\left(\begin{array}{c}
N \\
2
\end{array}\right)-\left(\begin{array}{c}
n_{1} \\
2
\end{array}\right)+\sum_{i \geqslant 2} n_{i}\left(\begin{array}{c}
m_{i}+1 \\
2
\end{array}\right)+1
$$

and applying (26) gives

$$
\tau_{1}^{\prime}(\mathcal{S}) \leqslant\left(\begin{array}{c}
N \\
2
\end{array}\right)-\left(\begin{array}{c}
n_{1} \\
2
\end{array}\right)+\left(\begin{array}{c}
N-n_{1}+1 \\
2
\end{array}\right)+1 .
$$

It may be seen that the right hand side of this simplifies to $N\left(N-n_{1}\right)+1$ as required. Equality occurs when $d=N-n_{1}$ and we have equality in (26), which is equivalent to the conditions given in (i).

In the case $m_{1}=2$, simplifying the definition of $\tau_{2}^{\prime}(\mathcal{S})$ gives

$$
\tau_{2}^{\prime}(\mathcal{S})=\left(\begin{array}{l}
N \\
2
\end{array}\right)+\sum_{i \geqslant 2} n_{i}\left(\begin{array}{c}
m_{i}+1 \\
2
\end{array}\right)+3+\epsilon,
$$

and after applying (26) we have

$$
\tau_{2}^{\prime}(\mathcal{S}) \leqslant\left(\begin{array}{l}
N \\
2
\end{array}\right)+\left(\begin{array}{c}
N-2 n_{1}+1 \\
2
\end{array}\right)+3+\epsilon .
$$

We must therefore show that

$$
\left(\begin{array}{l}
N \\
2
\end{array}\right)+\left(\begin{array}{c}
N-2 n_{1}+1 \\
2
\end{array}\right)+3 \leqslant N\left(N-n_{1}\right)+1
$$

Simplifying this gives

$$
\begin{gathered}
N(N-1)+\left(N-2 n_{1}+1\right)\left(N-2 n_{1}\right)+4 \leqslant 2 N\left(N-n_{1}\right) \\
4 \leqslant 2 N n_{1}-4 n_{1}^{2}+2 n_{1} \\
2 \leqslant n_{1}\left(N-2 n_{1}+1\right) .
\end{gathered}
$$

The result now follows from $n_{1} \geqslant 2$ and $N \geqslant n_{1} m_{1}=2 n_{1}$, and equality occurs exactly in case (ii).

When $m_{1}=3$, after simplifying the definition of $\tau_{3}^{\prime}(\mathcal{S})$ and dropping the $\epsilon$ term, we must show that

$$
\left(\begin{array}{l}
N \\
2
\end{array}\right)+6+\sum_{i \geqslant 2} n_{i}\left(\begin{array}{c}
m_{i}+1 \\
2
\end{array}\right) \leqslant\left(\begin{array}{l}
N \\
2
\end{array}\right)+6+\left(\begin{array}{c}
N-3 n_{1}+1 \\
2
\end{array}\right) \leqslant N\left(N-n_{1}\right),
$$

where the first inequality is (26). Simplifying this gives

$$
\begin{aligned}
N(N-1)+12+\left(N-3 n_{1}+1\right)\left(N-3 n_{1}\right) & \leqslant 2 N\left(N-n_{1}\right) \\
12 & \leqslant n_{1}\left(4 N-9 n_{1}+3\right) .
\end{aligned}
$$


We have $n_{1} \geqslant 2$ and $N \geqslant 3 n_{1}$, so that $N \geqslant 6$ and $4 N-9 n_{1}+3 \geqslant N+3 \geqslant 9$ as required.

\section{Acknowledgements}

We would like to thank Nicolas Bergeron for helpful discussions. The first author was supported by the NSF under grant no. 1501230, and under grant no. 1440140 while in residence at MSRI in Berkeley, California, during the spring of 2017. He was also supported as the Neil Chriss and Natasha Herron Chriss Founders' Circle Member while at the IAS in 2017-18. The second author was supported by NSF grant no. 1501882 and a Sloan Fellowship.

\section{Appendix A. Bounds for fixed vectors in representations of $\mathbf{G L}_{d}$}

In this section we prove bounds for the dimension of the vectors in an irreducible representation of $\mathrm{GL}_{d}$ that are invariant under a congruence subgroup, with no assumption on $d$ or the residue characteristic, by applying results of Lapid [18] on the support of Whittaker functions for supercuspidal representations. These results were used in the proof of Lemma 5.2 (thus in the proof of Proposition 5.1) when $m_{1}=2,3$.

Let $F$ be a $p$-adic field. Let $R$ be the ring of integers of $F, \varpi$ a uniformizer, $k$ the residue field, and $q$ its cardinality. Write $v: F^{\times} \rightarrow \mathbb{Z}$ for the additive valuation such that $v(\varpi)=1$. Let $d \geqslant 2$, and let $G=\operatorname{GL}_{d}(F)$. Let $N, A$, and $K=\mathrm{GL}_{d}(R)$ be the usual upper triangular unipotent, diagonal, and maximal compact subgroups of $G$, respectively. For $n \geqslant 0$ let $K(n)$ be the level $\varpi^{n}$ principal congruence subgroup of $K$ consisting of matrices which reduce to the identity matrix modulo $\varpi^{n}$.

We first prove the following result, which bounds $\operatorname{dim} \pi^{K(n)}$ for $\pi$ supercuspidal, before using it to deduce a bound for a general $\pi$ in Corollary A.3. Note that the constant $c$ in Theorem A.2 can be made explicit, which implies that the constants $C$ in Lemma A.1 and Corollary A.3 can be also.

LEMMA A.1. There is a constant $C$ depending only on $d$ such that for any $n \geqslant 1$ and any irreducible supercuspidal representation $\pi$ of $G$, we have $\operatorname{dim} \pi^{K(n)} \leqslant$ $C n^{d-1} q^{n d(d-1) / 2}$.

REMARK. We may obtain a uniform bound on $\operatorname{dim} \pi^{K(n)}$ of order $p^{\left(d^{2}-1\right) n}$ using the Plancherel theorem. Indeed, if we let $Z_{K}$ be the center of $K$, and $\omega$ be the central character of $\pi$, we may define $f$ to be the function supported on $Z_{K} K(n)$ and given by $f(z k)=\omega^{-1}(z)$. Applying the Plancherel theorem to $f$ 
gives $\operatorname{dim} \pi^{K(n)} \leqslant d(\pi)^{-1} \operatorname{vol}\left(Z_{K} K(n)\right)^{-1}$ for any Haar measure on $G$ and for any supercuspidal $\pi$, where $d(\pi)$ is the formal degree of $\pi$. By normalizing Haar measure, we can arrange that $d(\pi)$ is a positive integer, which gives $\operatorname{dim} \pi^{K(n)} \ll$ $q^{\left(d^{2}-1\right) n}$. This is considered a trivial bound. On the other hand, for a fixed $\pi$ (either supercuspidal or any generic representation of $G$ ), the asymptotic growth of $\operatorname{dim} \pi^{K(n)}$ is well known to be of order $q^{n d(d-1) / 2}$. (Such an asymptotic growth is known for general reductive $p$-adic groups either by character expansion or by a building argument [21, Theorem 8.5].) So the bound of Lemma A.1 is close to optimal (and more than enough for our global application). On a general reductive group, it is an interesting question whether a uniform bound can be established to the same order as the bound for an individual representation.

Proof of Lemma A.1. For all $n \geqslant 1$, define $A(n)$ to be the subset of $A$ given by

$$
A(n)=\left\{\operatorname{diag}\left(t_{1}, \ldots, t_{d}\right) \in A:-n \leqslant v\left(t_{i} / t_{i+1}\right) \leqslant n, i=1, \ldots, d-1\right\} .
$$

Let $\psi_{0}$ be an additive character of $F$ with conductor $R$, and let $\psi$ be the character of $N$ defined by $\psi(n)=\psi_{0}\left(n_{1,2}+\cdots+n_{d-1, d}\right)$. For a generic irreducible representation $\pi$ of $G$ let $\mathcal{W}(\pi)$ be the Whittaker model of $\pi$ with respect to $\psi$. In [18, Theorem 2.1], Lapid proves the following.

THEOREM A.2 (Lapid). There exists a constant $c=c(d)>0$ with the following property. Let $\pi$ be an irreducible supercuspidal representation of $G$ with Whittaker model $\mathcal{W}(\pi)$, and $n \geqslant 1$. Then the support of any $W \in \mathcal{W}(\pi)^{K(n)}$ is contained in $N A(c n) K$.

Let $G_{0}=\mathrm{GL}_{d-1}(F)$, embedded in $G$ as the upper-left block. Let $N_{0}, K_{0}$, and $K_{0}(n)$ be the corresponding subgroups of $G_{0}$. Define $T_{0}$ to be the diagonal subgroup of $G_{0}$, and for $n \geqslant 1$, define $T_{0}(n)$ to be $T_{0} \cap A(n)$. We are going to deduce Lemma A.1 from Theorem A.2 by combining it with the result of Bernstein-Zelevinsky that the restriction map $\mathcal{W}(\pi) \rightarrow C\left(G_{0}\right)$ is injective.

Let $W \in \mathcal{W}(\pi)^{K(n)}$, and let $W_{0}$ be its restriction to $G_{0}$. Theorem A.2 implies that $W_{0}$ is supported on $N_{0} T_{0}(\mathrm{cn}) K_{0}$, and it is left equivariant under $N_{0}$ and right invariant under $K_{0}(n)$. Let $\mathcal{V}_{0}$ be the space of functions on $N_{0} T_{0}(c n) K_{0}$ satisfying these conditions. Any $V \in \mathcal{V}_{0}$ is determined by its values on $T_{0}(c n) K_{0} / K_{0}(n)$, and $\operatorname{dim} \mathcal{V}_{0}$ is the number of left $N_{0}$-orbits on $T_{0}(c n) K_{0} / K_{0}(n)$. If $T_{0, c}$ is the maximal compact subgroup of $T_{0}$, there is a surjective map $T_{0}(c n) K_{0} / K_{0}(n) \rightarrow$ $T_{0}(c n) / T_{0, c}$ that is constant on the orbits of $N_{0}$. The fiber of this map above $t \in T_{0}(\mathrm{cn})$ is naturally identified with $K_{0} / K_{0}(n)$, and under this identification the orbits of $N_{0}$ on the fiber are the same as those of $N_{0} \cap K_{0}$ on $K_{0} / K_{0}(n)$. It follows that the number of $N_{0}$-orbits on $T_{0}(c n) K_{0} / K_{0}(n)$ is equal to the product 
of \# $T_{0}(c n) / T_{0, c}$ and \#( $\left.N_{0} \cap K_{0}\right) \backslash K_{0} / K_{0}(n)$. We have

$$
\# T_{0}(c n) / T_{0, c}=(2 c n+1)^{d-1} \leqslant C(d) n^{d-1}
$$

for some $C(d)>0$, and $\#\left(N_{0} \cap K_{0}\right) \backslash K_{0} / K_{0}(n) \leqslant q^{n d(d-1) / 2}$, so $\operatorname{dim} \mathcal{V}_{0} \leqslant$ $C(d) n^{d-1} q^{n d(d-1) / 2}$. As the map $\mathcal{W}(\pi)^{K(n)} \rightarrow \mathcal{V}_{0}$ given by restriction to $G_{0}$ is injective, this implies the Lemma.

COROllary A.3. There is a constant $C$ depending only on $d$ such that for any $n \geqslant 1$ and any irreducible representation $\pi$ of $G$, we have $\operatorname{dim} \pi^{K(n)} \leqslant$ $C n^{d-1} q^{n d(d-1) / 2}$.

Proof. There is a standard parabolic $P$ with Levi $\prod_{i=1}^{k} \mathrm{GL}_{d_{i}}$, and irreducible supercuspidal representations $\sigma_{i}$ of $\mathrm{GL}_{d_{i}}$, such that $\pi$ embeds in $\operatorname{Ind}_{P}^{G}\left(\sigma_{1} \times \cdots \times \sigma_{k}\right)$. We have

$$
\begin{aligned}
\operatorname{dim} \pi^{K(n)} & \leqslant \operatorname{dim} \operatorname{Ind}_{P}^{G}\left(\sigma_{1} \times \cdots \times \sigma_{k}\right)^{K(n)} \\
& =\#(P \cap K \backslash K / K(n)) \prod_{i=1}^{k} \operatorname{dim} \sigma_{i}^{K_{i}(n)},
\end{aligned}
$$

where $K_{i}(n)$ are the level $\varpi^{n}$ principal congruence subgroups of $\mathrm{GL}_{d_{i}}$. Applying Lemma A.1 gives

$$
\operatorname{dim} \pi^{K(n)} \leqslant C(d) \#(P \cap K \backslash K / K(n)) n^{d-1} q^{n \sum\left(\begin{array}{c}
d_{i} \\
2
\end{array}\right),}
$$

and the bound $\#(P \cap K \backslash K / K(n)) \leqslant C q^{n \operatorname{dim}(G / P)}$ finishes the proof.

We may rewrite Corollary A.3 in a form which is less sharp, but better suited to the proof of our main theorem.

COROLLARY A.4. If $\pi$ is an irreducible admissible representation of $G$, then for every $\epsilon>0$ there is a constant $C(\epsilon, q)>0$ such that

$$
\operatorname{dim} \pi^{K(n)} \leqslant C(\epsilon, q) q^{(d(d-1) / 2+\epsilon) n} .
$$

Moreover, for any $\epsilon>0$ there is $q(\epsilon)>0$ such that we may take $C(\epsilon, q)=1$ for all $q>q(\epsilon)$.

\section{Appendix B. Bounds for fixed vectors in representations of $\mathrm{GL}_{3}$}

Let $F$ be a $p$-adic field. Throughout this section we assume $p \neq 2,3$. Let $R$ be the ring of integers of $F, \varpi$ a uniformizer, $k$ the residue field, and $q$ its 
cardinality. Write $v: F^{\times} \rightarrow \mathbb{Z}$ for the additive valuation such that $v(\varpi)=1$. Let $G=\mathrm{GL}_{3}(F), K=\mathrm{GL}_{3}(R)$, and $A=M_{3}(R)$. Let $K_{j}$ be the subgroup of $K$ containing all elements congruent to 1 modulo $\varpi^{j}$. Put $U_{j}=1+\pi^{j} R$, a subgroup of $F^{\times}$. The following result was used in an earlier version of this paper as a substitute for the results of Appendix A. We have decided to leave it in, as it may be of independent interest.

THEOREM B.1. Assume $p \neq 2$,3. If $\pi$ is an irreducible supercuspidal representation of $G$, then

$$
\operatorname{dim} \pi^{K_{n}} \leqslant 9 n^{2} q^{4 n}(1+1 / q)^{3} .
$$

The proof uses the construction of supercuspidal representations of $\mathrm{GL}_{n}(F)$ by Howe in [15], in the case $n=3$. It was shown in [26] that these exhaust all supercuspidal representations of $G$ when $p$ is not 3 .

REMARK. We rely on [15], where it is essential to assume $p \neq 3$ (or more generally $p \nmid r$ if we study $\mathrm{GL}_{r}$ ). It may be superfluous to require $p \neq 2$, but as $p$ is odd in [15], we keep this assumption. When $p \in\{2,3\}$, one could try to adapt our argument using the construction of supercuspidals in [10] or [7], but we have not investigated this as Appendix A gives the desired result for our purpose for all $p$ via a simpler approach.

B.1. An overview of Howe's construction. We now describe the construction of Howe in more detail, including the features we shall use to prove Theorem B.1. Howe's representations $V\left(\psi^{\prime}\right)$ are parametrized by a degree 3 extension $F^{\prime} / F$ and a character $\psi^{\prime}$ of $F^{\prime x}$, satisfying a condition called admissibility. Fix such an $F^{\prime}$ and $\psi^{\prime}$, and let $R^{\prime}, \varpi^{\prime}$, and $k^{\prime}$ be the ring of integers, uniformizer, and residue field of $F^{\prime}$. Write $N=N\left(F^{\prime} / F\right)$ for the norm map from $F^{\prime}$ to $F$. Choose a basis for $R^{\prime}$ as a free $R$-module, which defines an embedding $F^{\prime} \subset M_{3}(F)$. We shall identify $F^{\prime}$ with a subalgebra of $M_{3}(F)$ from now on. We define the order $A^{\prime}=\bigcap_{x \in F^{\prime} \times} x A x^{-1}$, which is characterized as the set of matrices $M$ such that $M \varpi^{\prime i} R^{3} \subset \varpi^{\prime i} R^{3}$ for all $i$. We define $K^{\prime}=\bigcap_{x \in F^{\prime} \times} x K x^{-1}=A^{\prime} \cap \mathrm{GL}_{3}(R)$, which is the subgroup of matrices preserving the lattices $\varpi^{\prime i} R^{3}$. For $i \geqslant 1$ we define $K_{i}^{\prime}=1+\varpi^{\prime i} A^{\prime}$ and $U_{i}^{\prime}=1+\varpi^{\prime i} R^{\prime}$. Let $j$ be the conductor of $\psi^{\prime}$, that is the minimal $j$ such that $\psi^{\prime}$ is trivial on $U_{j}^{\prime}$. The admissibility condition placed on $\psi^{\prime}$ implies that $j \geqslant 1$.

In [15, Lemma 12], Howe constructs a representation $W\left(\psi^{\prime}\right)$ of $K^{\prime} F^{\prime \times}$, and defines the supercuspidal representation $V\left(\psi^{\prime}\right)$ associated to $F^{\prime}$ and $\psi^{\prime}$ to be the compact induction of $W\left(\psi^{\prime}\right)$ to $G$. (Lemma 12 only defines $W\left(\psi^{\prime}\right)$ as a 
representation of $K^{\prime}$, but it can be extended to $K^{\prime} F^{\prime \times}$ by the remarks at the start of [15, Theorem 2].) We know that $\operatorname{dim} \pi^{K_{n}}$ is at most $\operatorname{dim} W\left(\psi^{\prime}\right)$ times the number of double cosets of the form $K^{\prime} F^{\prime \times} g K_{n}$ that support $K_{n}$-invariant vectors, that is such that $W\left(\psi^{\prime}\right)^{g K_{n} g^{-1} \cap K^{\prime} F^{\prime \times}} \neq 0$. Bounding $\operatorname{dim} W\left(\psi^{\prime}\right)$ is easy, while bounding the number of these double cosets requires a feature of $W\left(\psi^{\prime}\right)$ from Howe's paper that we now describe.

We first assume that $j \geqslant 2$. The representation $W\left(\psi^{\prime}\right)$ is trivial on $K_{j}^{\prime}$, and because $K_{j-1}^{\prime} / K_{j}^{\prime}$ is abelian, $\left.W\left(\psi^{\prime}\right)\right|_{K_{j-1}^{\prime}}$ decomposes into characters. Howe defines a character $\psi$ of $K_{j-1}^{\prime} / K_{j}^{\prime}$ by taking the natural extension of $\psi^{\prime}$ from $U_{j-1}^{\prime}$, and shows that $\left.W\left(\psi^{\prime}\right)\right|_{K_{j-1}^{\prime}}$ contains exactly the characters lying in the $K^{\prime}$-orbit of $\psi$ for the natural action of $K^{\prime}$ on $\widehat{K_{j-1}^{\prime} / K_{j}^{\prime}}$.

We use this fact to control those $g$ supporting invariant vectors by observing that if $W\left(\psi^{\prime}\right)^{g K_{n} g^{-1} \cap K^{\prime} F^{\prime x}} \neq 0$, then $W\left(\psi^{\prime}\right)^{g K_{n} g^{-1} \cap K_{j-1}^{\prime}} \neq 0$. However, if $g \in K \lambda(\varpi) K$ with $\lambda \in X_{*}(T)$ too large, then $g K_{n} g^{-1} \cap K_{j-1}^{\prime}$ will contain the intersection of $K_{j-1}^{\prime}$ with a unipotent subgroup of $G$, and this will turn out to be incompatible with the description of $\left.W\left(\psi^{\prime}\right)\right|_{K_{j-1}^{\prime}}$. In the case $j=1, F^{\prime}$ is unramified over $F$ and $W\left(\psi^{\prime}\right)$ is inflated from a cuspidal representation of $K^{\prime} / K_{1}^{\prime} \simeq \mathrm{GL}_{3}(k)$, and we may use this to argue in a similar way.

In the case of $\mathrm{GL}_{3}$, Howe's construction may be naturally divided into the cases where $F^{\prime} / F$ is ramified or unramified. We shall therefore divide our proof into these two cases, after introducing some more notation and defining the character $\psi$.

B.2. The character $\psi$. We now assume that $j \geqslant 2$, and define the character $\psi$ of $K_{j-1}^{\prime} / K_{j}^{\prime}$ that appears in the description of $\left.W\left(\psi^{\prime}\right)\right|_{K_{j-1}^{\prime}}$.

Let $B^{\prime}$ be the group of prime to $p$ roots of unity in $F^{\prime x}$, which is naturally identified with $k^{\prime \times}$. Let $C^{\prime}$ be the group generated by $B^{\prime}$ and $\varpi^{\prime}$. Let $\langle$,$\rangle be$ the pairing $\langle S, T\rangle=\operatorname{tr}(S T)$ on $M_{3}(F)$. Let $\chi$ be a character of $F$ of conductor $R$, which defines an isomorphism $\theta: M_{3}(F) \rightarrow \widehat{M_{3}(F)}$ by $\theta(S)(T)=\chi(\langle S, T\rangle)$. Let $e$ denote the degree of ramification of $F^{\prime} / F$. Because the dual lattice to $A^{\prime}$ under $\langle$,$\rangle is \varpi^{\prime 1-e} A^{\prime}$ by [15, Lemma 2], the map $\theta$ gives an isomorphism between the character group of $\varpi^{\prime i-1} A^{\prime} / \varpi^{\prime i} A^{\prime}$ and $\varpi^{-i-e+1} A^{\prime} / \varpi^{-i-e+2} A^{\prime}$. We may combine $\theta$ with the isomorphism $K_{j-1}^{\prime} / K_{j}^{\prime} \simeq \varpi^{\prime j-1} A^{\prime} / \varpi^{\prime j} A^{\prime}$ to obtain $\mu: \widehat{K_{j-1}^{\prime} / K_{j}^{\prime}} \rightarrow$ $\varpi^{-j-e+1} A^{\prime} / \varpi^{-j-e+2} A^{\prime}$. If $\mu(\varphi)=y+\varpi^{-j-e+2} A^{\prime}$, we say that $y$ represents $\varphi$. If $\varphi$ has a representative $y \in F^{\prime x}$, we see that $\varphi$ also has a unique representative $c \in C^{\prime}$, which is called the standard representative of $\varphi$.

The map $\theta$ restricts to a map $F^{\prime} \rightarrow \widehat{F}^{\prime}$, which is also given by $\theta(x)(y)=\chi\left(\operatorname{tr}_{F^{\prime} / F} x y\right)$. We may combine $\theta$ with the isomorphism $U_{j-1}^{\prime} / U_{j}^{\prime} \simeq$ 
$\varpi^{\prime j-1} R^{\prime} / \varpi^{\prime j} R^{\prime}$ to obtain $\mu^{\prime}: \widehat{U_{j-1}^{\prime} / U_{j}^{\prime}} \rightarrow \varpi^{-j-e+1} R^{\prime} / \varpi^{-j-e+2} R^{\prime}$. If $\mu^{\prime}(\varphi)=y+\varpi^{-j-e+2} R^{\prime}$, we say that $y$ represents $\varphi$. We see that a nontrivial $\varphi$ has a unique representative $c \in C^{\prime}$, which is called the standard representative of $\varphi$.

We define $\psi$ by taking the standard representative $c$ for $\psi^{\prime}$ on $U_{j-1}^{\prime}$, and letting $\psi$ be the character represented by $c$. If we let $\mathrm{Ad}^{*}$ denote the natural action of $K^{\prime}$ on $\widehat{K_{j-1}^{\prime} / K_{j}^{\prime}}$, given explicitly by $\left[\operatorname{Ad}^{*}(k) \psi\right](g)=\psi\left(k^{-1} g k\right)$, then [15, Lemma 12] states that $\left.W\left(\psi^{\prime}\right)\right|_{K_{j-1}^{\prime}}$ contains exactly the characters in $\operatorname{Ad}^{*}\left(K^{\prime}\right) \psi$.

B.3. Reduction to the case $\boldsymbol{c} \notin \boldsymbol{F}$. We may carry out the argument sketched in Section B.1 once we have reduced to the case where either $j=1$ or $c \notin F$. We perform this reduction by observing that if $j \geqslant 2$ and $c \in F$, then $V\left(\psi^{\prime}\right)$ is a twist of $V\left(\psi_{1}\right)$ for some $\psi_{1}$ of smaller conductor. Indeed, by [15, Lemma 11], if $c \in F$ then we may write $\psi^{\prime}=\psi_{1} \psi_{2}$, where $\psi_{1}$ is trivial on $U_{j-1}^{\prime}$ and $\psi_{2}=\psi^{\prime \prime} \circ N\left(F^{\prime} / F\right)$ for some character $\psi^{\prime \prime}$ of $F^{\times}$.

LEMMA B.2. We have $V\left(\psi^{\prime}\right)=V\left(\psi_{1}\right) \otimes \psi^{\prime \prime} \circ$ det.

Proof. This follows by examining the construction of $W\left(\psi^{\prime}\right)$ in [15, Lemma 12]. In the case $c \in F$, the groups $H_{i}$ defined by Howe are equal to $K_{i}^{\prime}$, and the group $\mathrm{GL}_{l}\left(F^{\prime \prime}\right)$ appearing in the proof of [15, Lemma 12] is equal to $\mathrm{GL}_{3}(F)$. Howe constructs $W\left(\psi^{\prime}\right)$ by taking the representation $W\left(\psi_{1}\right)$ of $K^{\prime}$ associated to $\psi_{1}$ (which he denotes $W^{\prime \prime}\left(\psi_{1}^{\prime}\right)$, and whose construction can be assumed as $\psi_{1}$ has smaller conductor) and forming the twist $W\left(\psi_{1}\right) \otimes \psi^{\prime \prime} \circ$ det. He then obtains $W\left(\psi^{\prime}\right)$ by applying the correspondence of [15, Theorem 1] to $W\left(\psi_{1}\right) \otimes \psi^{\prime \prime} \circ$ det, which in this case is trivial so that $W\left(\psi^{\prime}\right)=W\left(\psi_{1}\right) \otimes \psi^{\prime \prime} \circ$ det. As $V\left(\psi^{\prime}\right)$ and $V\left(\psi_{1}\right)$ are the inductions of $W\left(\psi^{\prime}\right)$ and $W\left(\psi_{1}\right)$, the lemma follows.

The next lemma shows that it suffices to consider $V\left(\psi_{1}\right)$.

LEMMA B.3. We have $\operatorname{dim} V\left(\psi^{\prime}\right)^{K_{n}} \leqslant \operatorname{dim} V\left(\psi_{1}\right)^{K_{n}}$.

Proof. Because $N\left(U_{i}^{\prime}\right)=U_{\lceil i / e\rceil}$, if a character $\varphi$ of $F^{\times}$has conductor $i+1$, then $\varphi \circ N\left(F^{\prime} / F\right)$ has conductor $e i+1$. Because $\psi^{\prime \prime} \circ N$ has conductor $j \geqslant 2$, this implies that there is some $i \geqslant 1$ such that $j=e i+1$ and $\psi^{\prime \prime}$ has conductor $i+1$.

If $n \geqslant i+1$ then $\operatorname{det} K_{n} \subset U_{i+1}$. This implies that $\psi^{\prime \prime} \circ \operatorname{det}$ is trivial on $K_{n}$, which gives the lemma. Suppose that $n \leqslant i$ and $V\left(\psi^{\prime}\right)^{K_{n}} \neq 0$. As the central character of $V\left(\psi^{\prime}\right)$ is $\left.\psi^{\prime}\right|_{F^{\times}}$, this implies that $\psi^{\prime}$ is trivial on $U_{n}$, and hence on $U_{i}$. As $\psi_{1}$ is trivial on $U_{j-1}^{\prime} \cap F=U_{i}$, this implies that $\psi_{2}=\psi^{\prime \prime} \circ N\left(F^{\prime} / F\right)$ is trivial 
on $U_{i}$. This implies that $\psi^{\prime \prime}$ is trivial on $U_{i}$, which contradicts it having conductor $i+1$.

By replacing $\psi^{\prime}$ with $\psi_{1}$, multiple times if necessary, we may assume for the rest of the proof that $j=1$ or $c \notin F$.

B.4. The unramified case. Here, the groups $K^{\prime}$ and $K_{j}^{\prime}$ are equal to $K$ and $K_{j}$, respectively, and so we omit the ' in this section. We also take $\varpi^{\prime}=\varpi$. The embedding $F^{\prime} \subset M_{3}(F)$ has the property that $R^{\prime}=F^{\prime} \cap M_{3}(R)$, so that it induces an embedding $k^{\prime} \subset M_{3}(k)$. It also satisfies $R^{\prime \times}=F^{\prime \times} \cap K$ and $U_{i}^{\prime}=F^{\prime \times} \cap K_{i}$.

We need to bound $\operatorname{dim} W\left(\psi^{\prime}\right)$, and the number of double cosets $K F^{\prime \times} g K_{n}$ such that $W\left(\psi^{\prime}\right)^{g K_{n} g^{-1} \cap K F^{\prime x}} \neq 0$, and we begin with the second problem. We note that $F^{\prime \times} \subset K Z$ in the unramified case, where $Z$ is the center of $G$, so that $K F^{\prime \times}=K Z$. The dimension of $W\left(\psi^{\prime}\right)^{g K_{n} g^{-1} \cap K Z}$ depends only on the double coset $K Z g K$. By the Cartan decomposition, we may therefore break the problem into finding those $\lambda \in X_{*}(T)^{+}$such that $Z K \lambda(\varpi) K$ supports invariant vectors, where $T$ is the diagonal torus in $G$, and then count the $\left(Z K, K_{n}\right)$-double cosets in a given $Z K \lambda(\varpi) K$. These steps are carried out by Lemmas B.4 and B.5. We write $\lambda \in X_{*}(T)$ as $\left(\lambda_{1}, \lambda_{2}, \lambda_{3}\right)$.

LEMMA B.4. If $\lambda \in X_{*}(T)^{+}$is such that $W\left(\psi^{\prime}\right)^{\lambda(\varpi) K_{n} \lambda(\varpi)^{-1} \cap K Z} \neq 0$, then $\max \left\{\lambda_{1}-\lambda_{2}, \lambda_{2}-\lambda_{3}\right\} \leqslant n-j$. (It follows that $j \leqslant n$.)

Proof. We will prove $\lambda_{1}-\lambda_{2} \leqslant n-j$ by contradiction; the argument for $\lambda_{2}-\lambda_{3}$ is exactly analogous.

First we treat the case $j>1$. The hypothesis implies that $W\left(\psi^{\prime}\right)^{\lambda(\varpi) K_{n} \lambda(\varpi)^{-1} \cap K_{j-1}}$ $\neq 0$, and we use the description of $W\left(\psi^{\prime}\right)$ restricted to $K_{j-1}$. We identify $K_{j-1} / K_{j} \simeq M_{3}(k)$. If $c=b \varpi^{-j}$ with $b \in B^{\prime}$, and we identify $b$ with an element of $k^{\prime \times} \subset M_{3}(k)$, then $\left.\psi\right|_{K_{j-1}}$ under this identification corresponds to the character of $M_{3}(k)$ given by $x \rightarrow \chi(\operatorname{tr}(b x) / \varpi)$.

If $\lambda_{1}-\lambda_{2} \geqslant n-j+1$, a simple calculation shows that the image of $\lambda(\varpi) K_{n} \lambda(\varpi)^{-1} \cap K_{j-1}$ in $K_{j-1} / K_{j} \simeq M_{3}(k)$ contains the subgroup

$$
Y=\left(\begin{array}{l}
* \\
*
\end{array}\right) \subset M_{3}(k) .
$$

There must be a character in the orbit $\operatorname{Ad}^{*} K(\psi)$ which is trivial on $Y$, which means that there is $k \in K$ such that $\operatorname{tr}(y \operatorname{Ad}(k) b)=0$ for all $y \in Y$. The annihilator 
of $Y$ under the trace pairing is

$$
Y^{\perp}=\left(\begin{array}{l}
* \\
* * * \\
* * *
\end{array}\right) \subset M_{3}(k),
$$

so that $\operatorname{Ad}(k) b \in Y^{\perp}$. Any $y \in Y^{\perp}$ has eigenvalues that lie in the quadratic extension of $k$, while the eigenvalues of $b$ lie in $k^{\prime}-k$ because $c \notin F$, which is a contradiction.

Next we consider the case $j=1$. In this case, the proof of [15, Lemma 12] states that $W\left(\psi^{\prime}\right)$ is inflated from a cuspidal representation of $\mathrm{GL}_{3}(k)$. If $\lambda_{1}-$ $\lambda_{2} \geqslant n$, then the image of $\lambda(\varpi) K_{n} \lambda(\varpi)^{-1} \cap K$ in $K / K_{1} \simeq \mathrm{GL}_{3}(k)$ contains the subgroup

$$
Y=\left(\begin{array}{lll}
1 & \\
* & 1 & \\
* & 1
\end{array}\right) \subset \mathrm{GL}_{3}(k) .
$$

However, a cuspidal representation cannot have any vectors invariant under $Y$, because then it would be a subrepresentation of a representation induced from a parabolic of type $(2,1)$.

LEMMA B.5. Let $\lambda \in X_{*}(T)^{+}$satisfy $\max \left\{\lambda_{1}-\lambda_{2}, \lambda_{2}-\lambda_{3}\right\} \leqslant n-j$ as in Lemma B.4. The number of $\left(Z K, K_{n}\right)$-double cosets in $Z K \lambda(\varpi) K$ is at most $q^{4 n-4 j}(1+1 / q)^{3}$.

Proof. Any double coset $Z K g K_{n}$ contained in $Z K \lambda(\varpi) K$ has a representative with $g \in \lambda(\varpi) K$. It may be seen that $\lambda(\varpi) k_{1}$ and $\lambda(\varpi) k_{2}$ represent the same double coset if and only if $k_{1} \in \lambda(\varpi)^{-1} K \lambda(\varpi) k_{2} K_{n}$, and so if we define $K_{\lambda}=$ $\lambda(\varpi)^{-1} K \lambda(\varpi) \cap K$ then the number of double cosets is equal to $\left|K_{\lambda} \backslash K / K_{n}\right|$.

If $j=n$ then $\lambda_{1}=\lambda_{2}=\lambda_{3}$ so $K_{\lambda}=K$, hence the lemma is trivial. So we may assume $j \leqslant n-1$. Then $K_{\lambda}$ contains any matrix $g=\left(g_{a, b}\right) \in M_{3}(R)$ such that $v\left(g_{2,1}\right) \geqslant n-j, v\left(g_{3,2}\right) \geqslant n-j, v\left(g_{3,1}\right) \geqslant 2 n-2 j$, and $v\left(g_{i, i}\right)=0$ for $1 \leqslant i \leqslant 3$ (the last condition ensures that $g \in K$ as $g \bmod \varpi$ is upper triangular). This implies that the image of $K_{\lambda}$ in $K / K_{n} \simeq \mathrm{GL}_{3}\left(R / \varpi^{n}\right)$ has cardinality at least $q^{5 n+4 j}(1-1 / q)^{3}$. Therefore

$$
\begin{aligned}
\left|K_{\lambda} \backslash K / K_{n}\right| & \leqslant\left|K / K_{n}\right| /\left|\operatorname{image}\left(K_{\lambda}\right)\right| \\
& \leqslant q^{9 n}\left(1-q^{-3}\right)\left(1-q^{-2}\right)\left(1-q^{-1}\right) / q^{5 n+4 j}(1-1 / q)^{3} \\
& =q^{4 n-4 j}\left(1+q^{-1}+q^{-2}\right)\left(1+q^{-1}\right) \leqslant q^{4 n-4 j}(1+1 / q)^{3} .
\end{aligned}
$$

Lemma B.4 implies that there are at most $n^{2}$ choices of $\lambda \in X_{*}^{+}(T) / X_{*}(Z)$ such that $K Z \lambda(\varpi) K$ supports invariant vectors, and combining this with Lemma B.5 
shows that there are at most $n^{2} q^{4 n-4 j}(1+1 / q)^{3}$ double cosets $K Z g K_{n}$ that support invariant vectors. This gives

$$
\operatorname{dim} V\left(\psi^{\prime}\right)^{K_{n}} \leqslant n^{2} q^{4 n-4 j}(1+1 / q)^{3} \operatorname{dim} W\left(\psi^{\prime}\right) .
$$

We now bound $\operatorname{dim} W\left(\psi^{\prime}\right)$. We first assume that $j \geqslant 2$. Our assumption that $c \notin F$ implies that the field $F^{\prime \prime}$ in [15, Lemma 12] is the same as $F^{\prime}$, and the groups $H_{i}$ are given by $H_{0}=R^{\prime \times}$ and $H_{i}=U_{i}^{\prime}$ for $i \geqslant 1$. Following the proof of that lemma, we see that $W\left(\psi^{\prime}\right)$ is the representation associated to the character $\psi^{\prime}$ on $R^{\prime \times}$ by [15, Theorem 1]. When $j$ is even, that theorem implies that $W\left(\psi^{\prime}\right)$ is the induction of a character of $R^{\prime \times} K_{j / 2}$ to $K$, so that $\operatorname{dim} W\left(\psi^{\prime}\right)=\left|K: R^{\prime \times} K_{j / 2}\right|$. We have $\left|K: R^{\prime \times} K_{j / 2}\right|=q^{3 j}(1-1 / q)\left(1-1 / q^{2}\right) \leqslant q^{3 j}$.

When $j$ is odd, we let $j=2 i+1$. The construction of $W\left(\psi^{\prime}\right)$ in this case is described on [15, p. 448], and is given by inducing a representation $J$ from $R^{\prime \times} K_{i}$ to $K$. The discussion on p. 448 implies that $J$ has the same dimension as the two representations denoted $V\left(\widetilde{\varphi}_{\widetilde{\mathcal{H}}}^{\prime \prime}\right)$ and $\underset{\widetilde{Z}}{V}(\psi)$ there, and Howe states that $\operatorname{dim} V\left(\psi_{\tilde{\mathcal{Z}}}\right)=$ $(\# \widetilde{\mathcal{H}} / \widetilde{\mathcal{Z}})^{1 / 2}$ for two groups $\widetilde{\mathcal{H}}$ and $\widetilde{\mathcal{Z}}$. Moreover, on p. 447 he states that $\widetilde{\mathcal{H}} / \widetilde{\mathcal{Z}} \simeq$ $\mathcal{H} / \mathcal{Z} \simeq K_{i} / U_{i}^{\prime} K_{i+1}$. As $i \geqslant 1$, we have $\operatorname{dim} J=\left|K_{i} / U_{i}^{\prime} K_{i+1}\right|^{1 / 2}=q^{3}$. We then have $\operatorname{dim} W\left(\psi^{\prime}\right)=q^{3}\left|K: R^{\prime \times} K_{i}\right|=q^{3} q^{6 i}(1-1 / q)\left(1-1 / q^{2}\right) \leqslant q^{6 i+3}=q^{3 j}$.

In the remaining case $j=1, W\left(\psi^{\prime}\right)$ is inflated from a cuspidal representation of $\mathrm{GL}_{3}(k)$. Such a cuspidal representation has dimension $\left(q^{2}-1\right)(q-1)$. Therefore $\operatorname{dim} W\left(\psi^{\prime}\right)=\left(q^{2}-1\right)(q-1) \leqslant q^{3}=q^{3 j}$.

In all cases we have verified $\operatorname{dim} W\left(\psi^{\prime}\right) \leqslant q^{3 j}$. Hence

$$
\operatorname{dim} V\left(\psi^{\prime}\right)^{K_{n}} \leqslant n^{2} q^{4 n-4 j}(1+1 / q)^{3} \cdot q^{3 j} \leqslant n^{2} q^{4 n}(1+1 / q)^{3} .
$$

B.5. The ramified case. In this case we must have $j \geqslant 2$. Moreover, $F^{\prime}$ is tamely ramified over $F$ (since $p \neq 3$ ) and generated by a cube root of a uniformizer $\varpi$ of $F$. Thus we may assume $\varpi^{\prime 3}=\varpi$. Choose our basis for $R^{\prime}$ as a free $R$-module to be $\left\{1, \varpi^{\prime}, \varpi^{\prime 2}\right\}$. With this choice, the image of $\varpi^{\prime}$ in $G$ is

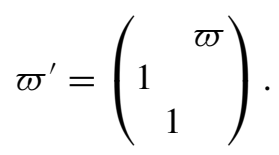

We see that $\varpi^{\prime i} A^{\prime}$ is given by

$$
\begin{aligned}
\varpi^{3 i} A^{\prime} & =\varpi^{i}\left(\begin{array}{ccc}
* & \varpi * & \varpi * \\
* & * & \varpi * \\
* & * & *
\end{array}\right), \\
\varpi^{3 i+1} A^{\prime} & =\varpi^{i}\left(\begin{array}{ccc}
\varpi * & \varpi * & \varpi * \\
* & \varpi * \varpi * \\
* & * & \varpi *
\end{array}\right),
\end{aligned}
$$




$$
\varpi^{3 i+2} A^{\prime}=\varpi^{i}\left(\begin{array}{ccc}
\varpi * & \varpi * & \varpi^{2} * \\
\varpi * & \varpi * & \varpi * \\
* & \varpi * & \varpi *
\end{array}\right)
$$

where the *'s lie in $R$. As $K^{\prime}=A^{\prime \times}, K^{\prime}$ is the lower triangular Iwahori subgroup.

The proof may be naturally broken into cases depending on the residue class of $j$ modulo 3. We may assume that $j \not \equiv 1$ (3), as in this case we have $c \in F$. Note that we are using our assumption that $\varpi^{13}=\varpi$ here.

As in the unramified case, we begin by observing that it suffices to bound $\operatorname{dim} W\left(\psi^{\prime}\right)$ and the number of double cosets $K^{\prime} F^{\prime \times} g K_{n}$ such that $W\left(\psi^{\prime}\right)^{g K_{n} g^{-1} \cap K^{\prime} F^{\times}} \neq 0$. This condition depends only on $K^{\prime} F^{\prime \times} g K$, and the following lemma gives a convenient set of representatives for these double cosets.

LEMmA B.6. If $\Sigma=\left\{\lambda \in X_{*}(T): \lambda_{1}+\lambda_{2}+\lambda_{3}=0\right\}$, we have $G=$ $\bigcup_{\lambda \in \Sigma} K^{\prime} F^{\prime \times} \lambda(\varpi) K$.

Proof. We use the Bruhat decomposition. Let $T^{1}$ and $N(T)$ be the maximal compact subgroup and normalizer of $T$. We define the Weyl group $W=N(T) / T$ and affine Weyl group $\widetilde{W}=N(T) / T^{1}$. We identify $W$ with the group of permutation matrices in $K$, and hence with a subgroup of $\widetilde{W}$. We then have $\widetilde{W} \simeq X_{*}(T) \rtimes W$, and $\widetilde{W}$ may be identified with matrices of the form $\lambda(\varpi) w$ with $\lambda \in X_{*}(T)$ and $w \in W$.

We have $\varpi^{\prime} \in N(T)$, and it may be seen that $\widetilde{W}=\left\langle\varpi^{\prime}\right\rangle \Sigma W$. Indeed, the action of $\varpi^{\prime}$ on $\widetilde{W} / W \simeq X_{*}(T)$ by left multiplication is given by

$$
\varpi^{\prime}\left(\lambda_{1}, \lambda_{2}, \lambda_{3}\right)=\left(\lambda_{3}+1, \lambda_{1}, \lambda_{2}\right),
$$

so every orbit contains a unique element of $\Sigma$. The Bruhat decomposition then gives

$$
G=\bigcup_{w \in \widetilde{W}} K^{\prime} w K^{\prime}=\bigcup_{\lambda \in \Sigma} K^{\prime}\left\langle\varpi^{\prime}\right\rangle \lambda(\varpi) W K^{\prime}=\bigcup_{\lambda \in \Sigma} K^{\prime} F^{\prime \times} \lambda(\varpi) K
$$

as required.

The next lemma bounds those $\lambda \in \Sigma$ such that $K^{\prime} F^{\prime \times} \lambda(\varpi) K$ supports invariant vectors.

LEMMA B.7. If $\lambda \in \Sigma$ satisfies $W\left(\psi^{\prime}\right)^{\lambda(\varpi) K_{n} \lambda(\varpi)^{-1} \cap K^{\prime} F^{\prime x}} \neq 0$, then

$$
\max \left\{\lambda_{1}-\lambda_{2}, \lambda_{2}-\lambda_{3}, \lambda_{3}-\lambda_{1}+1\right\} \leqslant n-i-1 \quad \text { if } j=3 i+2,
$$




$$
\max \left\{\lambda_{2}-\lambda_{1}, \lambda_{3}-\lambda_{2}, \lambda_{1}-\lambda_{3}-1\right\} \leqslant n-i-1 \text { if } j=3 i .
$$

In either case, summing the three bounds gives $j \leqslant 3 n-2$.

Proof. We may naturally identify $K^{\prime} / K_{1}^{\prime}$ and $K_{j-1}^{\prime} / K_{j}^{\prime}$ with $\left(k^{\times}\right)^{3}$ and $k^{3}$ using the coordinate entries in such a way that the adjoint action of $K^{\prime} / K_{1}^{\prime}$ on $K_{j-1}^{\prime} / K_{j}^{\prime}$ is given by

$$
\operatorname{Ad}\left(x_{1}, x_{2}, x_{3}\right)\left(y_{1}, y_{2}, y_{3}\right)=\left\{\begin{array}{l}
\left(x_{1} x_{2}^{-1} y_{1}, x_{2} x_{3}^{-1} y_{2}, x_{3} x_{1}^{-1} y_{3}\right), j \equiv 0(3), \\
\left(x_{1}^{-1} x_{2} y_{1}, x_{2}^{-1} x_{3} y_{2}, x_{3}^{-1} x_{1} y_{3}\right), j \equiv 2(3) .
\end{array}\right.
$$

Moreover, if $c$ is equal to $\varpi^{\prime-j-2} b$ with $b \in B^{\prime} \simeq k^{\times}$, then the character $\psi$ of $K_{j-1}^{\prime} / K_{j}^{\prime}$ is given by $\psi\left(y_{1}, y_{2}, y_{3}\right)=\chi\left(b\left(y_{1}+y_{2}+y_{3}\right) / \varpi\right)$. This implies that $\operatorname{Ad}^{*}(h) \psi$ is nontrivial on every coordinate subgroup in $K_{j-1}^{\prime} / K_{j}^{\prime} \simeq k^{3}$ for any $h \in K^{\prime}$.

If $W\left(\psi^{\prime}\right)^{\lambda(\varpi) K_{n} \lambda(\varpi)^{-1} \cap K^{\prime} F^{\prime x}} \neq 0$, then $W\left(\psi^{\prime}\right)^{\lambda(\varpi) K_{n} \lambda(\varpi)^{-1} \cap K_{j-1}^{\prime}} \neq 0$. Because $\left.W\left(\psi^{\prime}\right)\right|_{K_{j-1}^{\prime}}$ is a sum of characters of the form $\operatorname{Ad}^{*}(h) \psi$ with $h \in K^{\prime}$, one such character must be trivial on $\lambda(\varpi) K_{n} \lambda(\varpi)^{-1} \cap K_{j-1}^{\prime}$, which implies that the image of $\lambda(\varpi) K_{n} \lambda(\varpi)^{-1} \cap K_{j-1}^{\prime}$ in $K_{j-1}^{\prime} / K_{j}^{\prime}$ does not contain a coordinate subgroup. By combining the definition $K_{j-1}^{\prime}=1+\varpi^{\prime j-1} A^{\prime}$ with (B.1)-(B.3), we see that this implies the inequalities (B.4) and (B.5).

LemmA B.8. Let $\lambda \in \Sigma$ satisfy (B.4) or (B.5). The number of $\left(K^{\prime} F^{\prime \times}, K_{n}\right)$ double cosets in $K^{\prime} F^{\prime \times} \lambda(\varpi) K$ is at most $q^{3 n-2 i}(1+1 / q)^{3}$ when $j=3 i$, and $q^{3 n-2 i-2}(1+1 / q)^{3}$ when $j=3 i+2$.

Proof. Any double coset $K^{\prime} F^{\prime \times} g K_{n}$ contained in $K^{\prime} F^{\prime \times} \lambda(\varpi) K$ has a representative of the form $\lambda(\varpi) k$, and two elements $\lambda(\varpi) k_{1}$ and $\lambda(\varpi) k_{2}$ represent the same double coset if and only if $k_{2} \in \lambda(\varpi)^{-1} K^{\prime} F^{\prime \times} \lambda(\varpi) k_{1} K_{n}$. Therefore if we define $K_{\lambda}^{\prime}=\lambda(\varpi)^{-1} K^{\prime} \lambda(\varpi) \cap K$, the number of double cosets is bounded by $\left|K_{\lambda}^{\prime} \backslash K / K_{n}\right|$. It may be seen that $K_{\lambda}^{\prime}$ contains any matrix $g=\left(g_{a, b}\right) \in M_{3}(R)$ satisfying the conditions

$$
\begin{aligned}
v\left(g_{a, b}\right) & \geqslant \max \left\{\lambda_{b}-\lambda_{a}+1,0\right\}, \quad a<b \\
v\left(g_{i, i}\right) & =0, \quad 1 \leqslant i \leqslant 3 \\
v\left(g_{a, b}\right) & \geqslant \max \left\{\lambda_{b}-\lambda_{a}, 0\right\}, \quad a>b
\end{aligned}
$$

on the upper triangular, diagonal, and lower triangular entries, respectively. The reader should note that for each pair $a \neq b$, one may order $(a, b)$ so that the inequalities on $v\left(g_{a, b}\right)$ and $v\left(g_{b, a}\right)$ have the form $v\left(g_{a, b}\right) \geqslant c, v\left(g_{b, a}\right) \geqslant 0$ for some $c>0$. Moreover, the set of entries for which the inequality above reads $v\left(g_{a, b}\right) \geqslant 0$ 
form the unipotent radical of a Borel subgroup $B_{\lambda}$ containing the diagonal matrices. It follows that $K_{\lambda}^{\prime}$ lies between $B_{\lambda} \cap \mathrm{GL}_{3}(R)$ and $\left(B_{\lambda} \cap \mathrm{GL}_{3}(R)\right) K_{1}$.

We will divide the proof into six cases depending on the possibilities for $B_{\lambda}$. We treat one case in detail, and describe the modifications to be made in the others.

Case 1: $B_{\lambda}=\left(\begin{array}{r}* * * \\ * * \\ *\end{array}\right)$

In this case, the significant congruence conditions imposed on $g=\left(g_{a, b}\right) \in K_{\lambda}^{\prime}$ are

$$
v\left(g_{2,1}\right) \geqslant \lambda_{1}-\lambda_{2}, \quad v\left(g_{3,1}\right) \geqslant \lambda_{1}-\lambda_{3}, \quad \text { and } \quad v\left(g_{3,2}\right) \geqslant \lambda_{2}-\lambda_{3} .
$$

The image of $K_{\lambda}^{\prime}$ in $K / K_{n}$ therefore has cardinality at least $q^{9 n+2 \lambda_{3}-2 \lambda_{1}}(1-1 / q)^{3}$, and so as in Lemma B.5 we have $\left|K_{\lambda}^{\prime} \backslash K / K_{n}\right| \leqslant\left|K / K_{n}\right| /\left|\operatorname{image}\left(K_{\lambda}^{\prime}\right)\right| \leqslant$ $(1+1 / q)^{3} q^{2 \lambda_{1}-2 \lambda_{3}}$. If $j=3 i$ then Lemma B.7 gives $2 \lambda_{1}-2 \lambda_{3} \leqslant 2 n-2 i$ as required. If $j=3 i+2$, Lemma B.7 does not provide a strong enough bound for $\lambda_{1}-\lambda_{3}$, and so we instead observe that the image of $K_{\lambda}^{\prime}$ in $K / K_{n}$ contains those matrices satisfying

$$
v\left(g_{2,1}\right) \geqslant \lambda_{1}-\lambda_{2}, \quad v\left(g_{3,1}\right) \geqslant n, \quad \text { and } \quad v\left(g_{3,2}\right) \geqslant \lambda_{2}-\lambda_{3},
$$

with the other conditions unchanged. This group has cardinality at least $q^{8 n+\lambda_{3}-\lambda_{1}}(1-1 / q)^{3}$, and so $\left|K_{\lambda}^{\prime} \backslash K / K_{n}\right| \leqslant(1+1 / q)^{3} q^{n+\lambda_{1}-\lambda_{3}}$. Lemma B.7 gives

$$
\lambda_{1}-\lambda_{3}=\left(\lambda_{1}-\lambda_{2}\right)+\left(\lambda_{2}-\lambda_{3}\right) \leqslant 2 n-2 i-2,
$$

which gives the Lemma in this case.

In the other five cases, we may apply the same method to produce a bound of the form $\left|K_{\lambda}^{\prime} \backslash K / K_{n}\right| \leqslant(1+1 / q)^{3} q^{\tau}$, where $\tau$ depends on the residue class of $j$ modulo 3 . We describe the underlying recipe for finding $\tau$ in the case above, and then show what it gives in each remaining case. When $j \equiv 0$ (3), we added the right hand sides of (B.6), and the resulting expression $2\left(\lambda_{1}-\lambda_{3}\right)$ could be bounded using one application of Lemma B.7, which gave $\tau$. When $j \equiv 2$ (3), we modified the bound in (B.6) corresponding to the nonsimple positive root for $B_{\lambda}$ to obtain (B.7), added the right hand sides, and bounded the result using two applications of Lemma B.7 to give $\tau$.

We now find $\tau$ in the remaining five cases, and check that

$$
\tau \leqslant \begin{cases}3 n-2 i-2, & j=3 i+2, \\ 3 n-2 i, & j=3 i .\end{cases}
$$

Note that in some cases we may need to use the assumption that $n \geqslant 1$, which we are free to make. 
Case 2: $B_{\lambda}=\left(\begin{array}{c}* * * \\ * \\ * *\end{array}\right)$

The analog of (B.6) is

$$
v\left(g_{2,1}\right) \geqslant \lambda_{1}-\lambda_{2}, \quad v\left(g_{3,1}\right) \geqslant \lambda_{1}-\lambda_{3}, \quad v\left(g_{2,3}\right) \geqslant \lambda_{3}-\lambda_{2}+1,
$$

which is modified to $v\left(g_{2,1}\right) \geqslant n$. We have

$$
\begin{aligned}
2 \lambda_{1}-2 \lambda_{2}+1 \leqslant 2 n-2 i-1=\tau & \text { when } j=3 i+2, \\
n+\lambda_{1}-\lambda_{2}+1 \leqslant 3 n-2 i=\tau & \text { when } j=3 i .
\end{aligned}
$$

Case 3: $B_{\lambda}=\left(\begin{array}{rr}* & * \\ * & * \\ & *\end{array}\right)$

The analog of (B.6) is

$$
v\left(g_{1,2}\right) \geqslant \lambda_{2}-\lambda_{1}+1, \quad v\left(g_{3,1}\right) \geqslant \lambda_{1}-\lambda_{3}, \quad v\left(g_{3,2}\right) \geqslant \lambda_{2}-\lambda_{3},
$$

which is modified to $v\left(g_{3,2}\right) \geqslant n$. We have

$$
\begin{aligned}
2 \lambda_{2}-2 \lambda_{3}+1 \leqslant 2 n-2 i-1=\tau & \text { when } j=3 i+2, \\
n+\lambda_{2}-\lambda_{3}+1 \leqslant 3 n-2 i=\tau & \text { when } j=3 i .
\end{aligned}
$$

Case 4: $B_{\lambda}=\left(\begin{array}{c}* * \\ * \\ * * *\end{array}\right)$

The analog of (B.6) is

$$
v\left(g_{2,1}\right) \geqslant \lambda_{1}-\lambda_{2}, \quad v\left(g_{1,3}\right) \geqslant \lambda_{3}-\lambda_{1}+1, \quad v\left(g_{2,3}\right) \geqslant \lambda_{3}-\lambda_{2}+1,
$$

which is modified to $v\left(g_{2,3}\right) \geqslant n$. We have

$$
\begin{aligned}
2 \lambda_{3}-2 \lambda_{2}+2 \leqslant 2 n-2 i & =\tau \quad \text { when } j=3 i, \\
n+\lambda_{3}-\lambda_{2}+1 \leqslant 3 n-2 i-2=\tau & \text { when } j=3 i+2 .
\end{aligned}
$$

Case 5: $B_{\lambda}=\left(\begin{array}{lll}* & \\ * & * & * \\ * & *\end{array}\right)$

The analog of (B.6) is

$$
v\left(g_{1,2}\right) \geqslant \lambda_{2}-\lambda_{1}+1, \quad v\left(g_{1,3}\right) \geqslant \lambda_{3}-\lambda_{1}+1, \quad v\left(g_{3,2}\right) \geqslant \lambda_{2}-\lambda_{3},
$$


which is modified to $v\left(g_{1,2}\right) \geqslant n$. We have

$$
\begin{aligned}
2 \lambda_{2}-2 \lambda_{1}+2 \leqslant 2 n-2 i=\tau & \text { when } j=3 i, \\
n+\lambda_{2}-\lambda_{1}+1 \leqslant 3 n-2 i-2=\tau & \text { when } j=3 i+2 .
\end{aligned}
$$

Case 6: $B_{\lambda}=\left(\begin{array}{l}* \\ * * \\ * * *\end{array}\right)$

The analog of (B.6) is

$$
v\left(g_{1,2}\right) \geqslant \lambda_{2}-\lambda_{1}+1, \quad v\left(g_{1,3}\right) \geqslant \lambda_{3}-\lambda_{1}+1, \quad v\left(g_{2,3}\right) \geqslant \lambda_{3}-\lambda_{2}+1,
$$

which is modified to $v\left(g_{1,3}\right) \geqslant n$. We have

$$
\begin{aligned}
2 \lambda_{3}-2 \lambda_{1}+3 \leqslant 2 n-2 i-1=\tau & \text { when } j=3 i+2, \\
n+\lambda_{3}-\lambda_{1}+2 \leqslant 3 n-2 i=\tau & \text { when } j=3 i .
\end{aligned}
$$

There are at most $9 n^{2}$ choices of $\lambda \in \Sigma$ satisfying the bounds of Lemma B.7. Indeed, if $j=3 i+2$ then the Lemma gives $n-1 \geqslant \lambda_{1}-\lambda_{2}, \lambda_{2}-\lambda_{3} \geqslant-2 n+3$, and these two values determine $\lambda \in \Sigma$ uniquely. If $j=3 i$, we have $i \geqslant 1$ so the Lemma likewise gives $2 n-3 \geqslant \lambda_{1}-\lambda_{2}, \lambda_{2}-\lambda_{3} \geqslant-n+2$. Moreover, the bound of Lemma B.8 may be written as $q^{3 n-j+i}(1+1 / q)^{3}$ in either case $j=3 i$ or $j=3 i+2$. We therefore have at most $9 n^{2} q^{3 n-j+i}(1+1 / q)^{3}$ double cosets $K^{\prime} F^{\prime \times} g K_{n}$ that support invariant vectors, and

$$
\operatorname{dim} V\left(\psi^{\prime}\right)^{K_{n}} \leqslant 9 n^{2} q^{3 n-j+i}(1+1 / q)^{3} \operatorname{dim} W\left(\psi^{\prime}\right) .
$$

If $j$ is even, $W\left(\psi^{\prime}\right)$ is again obtained by inducing a character from $R^{\prime \times} K_{j / 2}^{\prime}$ to $K^{\prime}$, and we have $\operatorname{dim} W\left(\psi^{\prime}\right)=\left|K^{\prime}: R^{\prime \times} K_{j / 2}^{\prime}\right|=(1-1 / q)^{2} q^{j}$.

If $j$ is odd, set $j=2 l+1$. As before, Howe defines $W\left(\psi^{\prime}\right)$ to be the induction from $R^{\prime \times} K_{l}^{\prime}$ to $K^{\prime}$ of a representation of dimension $\left|K_{l}^{\prime}: U_{l}^{\prime} K_{l+1}^{\prime}\right|^{1 / 2}=q$. This gives $\operatorname{dim} W\left(\psi^{\prime}\right) \leqslant q\left|K^{\prime}: R^{\prime \times} K_{l}^{\prime}\right|=(1-1 / q)^{2} q^{2 l+1}=(1-1 / q)^{2} q^{j}$.

In either case, the bound $\operatorname{dim} W\left(\psi^{\prime}\right) \leqslant q^{j}$ gives

$$
\operatorname{dim} V\left(\psi^{\prime}\right)^{K_{n}} \leqslant 9 n^{2} q^{3 n-j+i}(1+1 / q)^{3} \cdot q^{j}=9 n^{2} q^{3 n+i}(1+1 / q)^{3} .
$$

If $j=3 i$ then the bound $j \leqslant 3 n-2$ from Lemma B.7 gives $i \leqslant n-1$, while if $j=3 i+2$ then $j \leqslant 3 n-2$ gives $i \leqslant n-2$. In either case, this completes the proof of Theorem B.1. 


\section{References}

[1] J. Arthur, The Endoscopic Classification of Representations, American Mathematical Society Colloquium Publications, 61 (American Mathematical Society, Providence, RI, 2013).

[2] N. Bergeron and L. Clozel, 'Spectre automorphe des variétés hyperboliques et applications topologiques', Astérisque 303 (2005), $\mathrm{xx}+218$.

[3] N. Bergeron, J. Millson and C. Moeglin, 'The Hodge conjecture and arithmetic quotients of complex balls', Acta Math. 216(1) (2016), 1-125.

[4] J. N. Bernstein, 'Le "centre" de Bernstein', in Representations of Reductive Groups Over a Local Field, Travaux en Cours (ed. P. Deligne) (Hermann, Paris, 1984), 1-32.

[5] I. N. Bernšteĭn, 'All reductive p-adic groups are of type I', Funkcional. Anal. i Priložen. 8(2) (1974), 3-6.

[6] A. Borel and N. Wallach, Continuous cohomology, discrete subgroups, and representations of reductive groups, 2nd edn, Mathematical Surveys and Monographs, 67 (American Mathematical Society, Providence, RI, 2000).

[7] C. J. Bushnell and P. C. Kutzko, The Admissible Dual of $\mathrm{GL}(N)$ via Compact Open Subgroups, Annals of Mathematics Studies, 129 (Princeton University Press, Princeton, NJ, 1993).

[8] K. Buzzard and T. Gee, 'The conjectural connections between automorphic representations and Galois representations', in Automorphic Forms and Galois Representations, Vol. 1, London Mathematical Society Lecture Note Series, 414 (Cambridge University Press, Cambridge, 2014), 135-187.

[9] A. Caraiani, 'Local-global compatibility and the action of monodromy on nearby cycles', Duke Math. J. 161(12) (2012), 2311-2413.

[10] H. Carayol, 'Représentations cuspidales du groupe linéaire', Ann. Sci. Éc. Norm. Supér. (4) 17(2) (1984), 191-225.

[11] L. Clozel, 'Motifs et formes automorphes: applications du principe de fonctorialité', in Automorphic Forms, Shimura Varieties, and L-Functions, Vol. I (Ann Arbor, MI, 1988), Perspectives on Mathematics, 10 (Academic Press, Boston, MA, 1990), 77-159.

[12] M. Cossutta and S. Marshall, 'Theta lifting and cohomology growth in $p$-adic towers', Int. Math. Res. Not. IMRN 2013(11) (2013), 2601-2623.

[13] D. L. de George and N. R. Wallach, 'Limit formulas for multiplicities in $L^{2}(\Gamma \backslash G)$ ', Ann. of Math. (2) 107(1) (1978), 133-150.

[14] S. S. Gelbart, Automorphic forms on adèle groups, Annals of Mathematics Studies, No. 83 (Princeton University Press, Princeton, NJ, 1975), University of Tokyo Press, Tokyo.

[15] R. E. Howe, 'Tamely ramified supercuspidal representations of $\mathrm{Gl}_{n}$ ', Pacific J. Math. 73(2) (1977), 437-460.

[16] T. Kaletha, A. Minguez, S. W. Shin and P.-J. White, 'Endoscopic classification of representations: Inner forms of unitary groups', Preprint, 2014, arXiv:1409.3731.

[17] A. W. Knapp, Representation Theory of Semisimple Groups, Princeton Landmarks in Mathematics, (Princeton University Press, Princeton, NJ, 2001).

[18] E. Lapid, 'On the support of matrix coefficients of supercuspidal representations of the general linear group over a local non-archimedean field', Preprint, 2018, arXiv: 1802.07154.

[19] S. Marshall, 'Endoscopy and cohomology growth on U(3)', Compos. Math. 150(6) (2014), 903-910.

[20] S. Marshall, 'Endoscopy and cohomology growth on a quasi-split $U(4)$ ', in Families of Automorphic Forms and the Trace Formula, Simons Symposia (Springer International Publishing Switzerland, 2016), 297-325. 
[21] R. Meyer and M. Solleveld, 'Characters and growth of admissible representations of reductive p-adic groups', J. Inst. Math. Jussieu 11(2) (2012), 289-331.

[22] C. Moeglin and D. Renard, 'Sur les paquets d'Arthur aux places réels, translation', in Geometric Aspects of the Trace Formula, Simons Symposia (Springer International Publishing, Switzerland, 2018), 299-320.

[23] C. Moeglin and J.-L. Waldspurger, Stabilisation de la formule des traces tordue, Vol. 1, Progress in Mathematics, 316 (Birkhäuser/Springer, Cham, 2016).

[24] C. Moeglin and J.-L. Waldspurger, Stabilisation de la formule des traces tordue, Vol. 2, Progress in Mathematics, 317 (Birkhäuser/Springer, Cham, 2016).

[25] C. P. Mok, 'Endoscopic classification of representations of quasi-split unitary groups', Mem. Amer. Math. Soc. 235(1108) (2015), vi+248.

[26] A. Moy, 'Local constants and the tame Langlands correspondence', Amer. J. Math. 108(4) (1986), 863-930.

[27] J. D. Rogawski, 'Analytic expression for the number of points mod p', in The Zeta Functions of Picard Modular Surfaces (Univ. Montréal, Montreal, QC, 1992), 65-109.

[28] P. Sarnak and X. X. Xue, 'Bounds for multiplicities of automorphic representations', Duke Math. J. 64(1) (1991), 207-227.

[29] G. Savin, 'Limit multiplicities of cusp forms', Invent. Math. 95(1) (1989), 149-159.

[30] D. A. Vogan Jr., 'Unitarizability of certain series of representations', Ann. of Math. (2) 120(1) (1984), 141-187.

[31] D. A. Vogan Jr. and G. J. Zuckerman, 'Unitary representations with nonzero cohomology', Compos. Math. 53(1) (1984), 51-90.

[32] N. R. Wallach, 'On the constant term of a square integrable automorphic form', in Operator Algebras and Group Representations, Vol. II (Neptun, 1980), Monographs and Studies in Mathematics, 18 (Pitman, Boston, MA, 1984), 227-237.

[33] X. X. Xue, 'On the first Betti numbers of hyperbolic surfaces', Duke Math. J. 64(1) (1991), $85-110$. 